\title{
On Trellis-Based Truncated-Memory Detection
}

\author{
Gianluigi Ferrari, Member, IEEE, Giulio Colavolpe, Associate Member, IEEE, and Riccardo Raheli, Member, IEEE
}

\begin{abstract}
We propose a general framework for trellis-based detection over channels with infinite memory. A general truncation assumption enables the definition of a trellis diagram, which takes into account a considered portion of the channel memory and possible coding memory at the transmitter side. It is shown that trellis-based maximum a posteriori (MAP) symbol detection algorithms, in the form of forward-backward (FB) algorithms, can be derived on the basis of this memory-truncation assumption. A general approach to the design of truncated-memory (TM) FB algorithms is proposed, and two main classes of algorithms, characterized by coupled and decoupled recursions, respectively, are presented. The complexity of the derived TM-FB algorithms is analyzed in detail. Moreover, it is shown that MAP sequence detection algorithms, based on the Viterbi algorithm, follow easily from one of the proposed classes. Looking backward at this duality between MAP symbol detection algorithms and MAP sequence detection algorithms, it is shown that previous solutions for one case can be systematically extended to the other case. The generality of the proposed framework is shown by considering various examples of stochastic channels. New detection algorithms, as well as generalizations of solutions previously published in the literature, are embedded in the proposed framework. The obtained results do suggest that the performance of the proposed detection algorithms ultimately depends on the truncation depth, almost regardless of the specific detection strategy.
\end{abstract}

Index Terms-Forward-backward (FB) algorithm, iterative detection, maximum a posteriori (MAP) sequence/symbol detection, memory truncation, trellis-based detection, Viterbi algorithm (VA).

\section{INTRODUCTION}

$\mathbf{T}$ HE study of detection strategies suitable for various possible transmission channels has received substantial attention in the communication theory literature [1]. Various approaches, each peculiar to a particular channel model, have been proposed. In particular, if the transmission channel is impaired by the presence of unknown parameters, a partial knowledge of these parameters, for example, statistical, may be available at the receiver. A general approach to approximately perform maximum-likelihood sequence detection (MLSD), based on a Viterbi algorithm (VA), in uncertain environments was proposed in [2], where the concept of per-survivor processing (PSP) was clearly formalized.

If the channel can be modeled as a finite state machine (FSM), i.e., its memory is finite, then a trellis diagram can be associated with it. However, if the channel memory is infinite, the

Paper approved by R. D. Wesel, the Editor for Coding and Communication Theory of the IEEE Communications Society. Manuscript received August 7, 2002; revised December 13, 2003 and November 23, 2004. This paper was presented in part at the IEEE Global Telecommunications Conference (GLOBECOM'03), San Francisco, CA, December 2003.

The authors are with the Department of Information Engineering, University of Parma, 43100 Parma, Italy (e-mail: gianluigi.ferrari@unipr.it; giulio.colavolpe@unipr.it; riccardo.raheli@unipr.it).

Digital Object Identifier 10.1109/TCOMM.2005.855008 application of the PSP approach is not unique: from an optimal tree-based search, a suboptimal trellis-based search must be derived. Two main approaches have been proposed. A first approach, considered in [2]-[4] for VA-based MLSD and extended in [5] and [6] to forward-backward (FB) algorithms, entails the arbitrary definition of a trellis diagram, where in each path, the infinite channel memory is recursively taken into account. A second approach, addressed in this paper, considers an a priori truncation of the channel memory, which naturally leads to the definition of a trellis diagram. In this case, all paths corresponding to the retained memory are taken into account. In the literature, truncated-memory (TM) detection has been widely considered. A few examples are given in the following.

- In the case of fading channels, linear predictive detection strategies [7]-[17] and noncoherent detection [18] have been considered.

- In the case of a phase-uncertain channel, a TM approach has been proposed in [19]-[22].

- In the case of a magnetic channel, MAP sequence detection algorithms based on a truncation of the autocorrelation function have been derived in [23].

In this paper, we propose a general framework for trellisbased TM detection. The memory-truncation assumption, introduced in general terms, is immediately applicable for detection over any channel with infinite memory. It is also shown that detection algorithms which perform maximum a posteriori (MAP) sequence detection can be easily derived from detection algorithms for MAP symbol detection. ${ }^{1}$ It follows that all the algorithmic solutions appeared in the literature for TM sequence detection based on a VA can be systematically extended to TM symbol detection based on an FB algorithm, and vice versa. Hence, the focus of this paper will be on FB algorithms.

Since an FB algorithm is based on two recursions, two classes of TM-FB algorithms are proposed. The first class is characterized by coupled recursions (CR), in the sense that the basic causal metric used in the forward recursion is also used in the backward recursion. The TM-FB algorithms in the second class are instead characterized by decoupled recursions (DR), in the sense that the metric used in the backward recursion is anticausal. In order to cope with the memory increase caused by truncation, state-reduction techniques recently proposed are employed for both classes of TM-FB algorithms [24]-[26]. A complexity analysis of TM-FB algorithms is presented, with particular attention to the case where state-reduction techniques are applied. It is shown that in order to obtain a comparable performance, the complexity required by TM-FB-DR algorithms is usually larger than that required by TM-FB-CR algorithms. In particular, the higher complexity of TM-FB-DR algorithms re-

\footnotetext{
${ }^{1}$ In the remainder of this paper, we refer to MAP symbol and MAP sequence detection simply as symbol and sequence detection, respectively.
} 


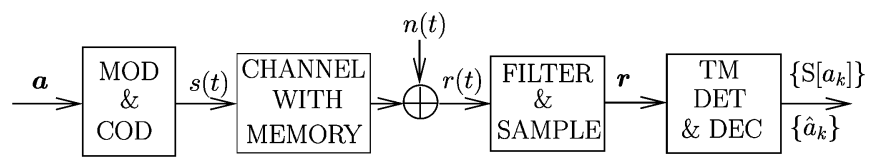

Fig. 1. Communication system.

sides in the completion rather than in the two recursions. ${ }^{2}$ However, reduced-state TM-FB-DR algorithms may exhibit implementation advantages, with respect to reduced-state TM-FB-CR algorithms, in terms of decoding latency.

The general formulation of TM-FB algorithms is specialized to two stochastic channels: a flat-fading channel and a phase-uncertain channel. Based on the specific channel model assumed in the design of TM-FB algorithms, several solutions are proposed. It is demonstrated that the truncation depth basically determines the performance. For the sake of simplicity, we consider only the case of linear modulations. The extension of all the proposed results to the case of continuous phase modulation (CPM) can be dealt with by considering, for example, the decomposition approach proposed in [27].

To summarize, in this paper, we will try to answer the following two key questions.

1) What are the advantages/disadvantages of the proposed algorithmic structures?

2) Given a particular algorithmic structure and complexity level, what is the impact of the specific TM detection strategy?

This paper is organized as follows. In Section II, we describe the considered communication system model. In Section III, we develop a general formulation relative to TM detection, focusing on TM-FB algorithmic structures and showing a simple extension to the case of a TM-VA. In Section IV, a complexity analysis of the proposed TM-FB algorithmic classes, and their reduced-state versions, is presented. In Section V, we show how the proposed TM-FB algorithms can be applied to a stochastic channel, and we consider two specific examples of such channels. In Section VI, numerical results are presented, and they are discussed in Section VII. Conclusions are finally drawn in Section VIII.

\section{COMMUniCATION System MODEL}

We consider a generic communication system, as depicted in Fig. 1. A sequence of independent and identically distributed $M$-ary information symbols $\left\{a_{k}\right\}$ are transmitted successively from epoch 0 to epoch $K-1$. A sequence of information symbols is denoted in vector notation as $\boldsymbol{a}_{k_{1}}^{k_{2}}=\left(a_{k_{1}}, a_{k_{1}+1}, \ldots, a_{k_{2}}\right), k_{2} \geq k_{1}$. For brevity, the entire sequence is denoted by $\boldsymbol{a}$. The information symbols feed an encoder/modulator, whose output is transmitted over a channel with memory and additive white Gaussian noise (AWGN). The encoder/modulator block can be described as a time-invariant FSM with state $\mu_{k}$. We denote the number of states of the encoder/modulator FSM as $\Omega$. The evolution of the encoder/modulator can be described by a trellis diagram. Upon

\footnotetext{
${ }^{2}$ We refer to the soft-output computation based on the combination of the metrics computed in the two recursions as completion.
}

the arrival of an information symbol $a_{k}$, the encoder/modulator FSM evolves according to a "next-state" function, such that $\mu_{k+1}=\mathrm{ns}\left(\mu_{k}, a_{k}\right)$. In correspondence with each transition in the trellis diagram, an output symbol $c_{k}$ is generated according to an "output" function $c_{k}=\operatorname{out}\left(\mu_{k}, a_{k}\right)$. We assume that the trellis is regular, in the sense that there are $M$ branches entering and departing from each state. For the sake of simplicity, we also assume that each of the $M$ branches entering a generic state $\mu_{k+1}$ is labeled by a different information symbol $a_{k}$-this is always true for the $M$ exiting branches. ${ }^{3}$

By means of a discretization process, the received signal $r(t)$ can be converted into a discrete sequence $\boldsymbol{r}=\boldsymbol{r}_{0}^{K-1}$. A sampling rate of one sample per signaling interval is considered for simplicity. We also assume that the considered system is causal, i.e., $p\left(\boldsymbol{r}_{0}^{k} \mid \boldsymbol{a}\right)=p\left(\boldsymbol{r}_{0}^{k} \mid \boldsymbol{a}_{0}^{k}\right)$. The last block in Fig. 1 implements $\mathrm{TM}$ detection/decoding. In the case of TM sequence detection based on the VA, an estimated information sequence $\left\{\hat{a}_{k}\right\}$ is generated. In the case of TM symbol detection, the ultimate goal of this block is the generation of the symbol a posteriori probability (APP), i.e., $P\left\{a_{k} \mid \boldsymbol{r}\right\}$, or a proportional quantity. In practice, a reliability value $S\left[a_{k}\right]$, which approximates the APP of each information symbol, is computed.

\section{TRELLIS-BASED TM DETECTION}

The detection approach proposed in the remainder of this paper is based on a memory-truncation assumption which can be stated in general terms, as it will be shown in the following. Intuitively, it is not practically important to take into account the entire channel memory, because a significant portion of it may be sufficient. This is confirmed by very good results obtained by applying state-reduction techniques [24], [25] to the algorithms derived in this paper. In the following, we first develop a general formulation for TM symbol detection, based on FB algorithms, and then extend it to the case of TM sequence detection, based on a VA.

\section{A. TM Symbol Detection: FB Algorithms}

We focus on the computation of a reliability value $S\left[a_{k}\right]$ by means of an FB algorithm [29]. We first develop the two classes of CR and DR TM-FB algorithms, based on a common memory-truncation assumption. In both cases, we start from the exact calculation of the APP, and then approximate it by a reliability value $S\left[a_{k}\right]$ which can be efficiently computed. Afterwards, we propose a heuristic generalization of TM-FB-CR algorithms suggested by the completion operation of TM-FB-DR algorithms. The starting point for the derivation of both classes of algorithms is the computation of the following APP:

$$
P\left\{a_{k} \mid \boldsymbol{r}\right\}=\sum_{\boldsymbol{a}: a_{k}} P\{\boldsymbol{a} \mid \boldsymbol{r}\} \propto \sum_{\boldsymbol{a}: a_{k}} p(\boldsymbol{r} \mid \boldsymbol{a}) P\{\boldsymbol{a}\}
$$

where the symbol $\propto$ denotes proportionality, and the notation $\boldsymbol{a}: a_{k}$ denotes all sequences $\boldsymbol{a}$ compatible with $a_{k}$.

${ }^{3}$ This assumption is valid for particular recursive encoders and simplifies the derivation. The final formulation is applicable, however, to any FSM model [28]. We will comment further on this aspect in Section III-A. 
1) TM-FB-CR Algorithms: Based on the causality assumption, one can write [28]

$$
\begin{aligned}
P\left\{a_{k} \mid \boldsymbol{r}\right\} \propto & \sum_{\boldsymbol{a}_{0}^{k-1}} \sum_{\mu_{k}} p\left(\boldsymbol{r}_{k+1}^{K-1} \mid \boldsymbol{r}_{0}^{k}, \boldsymbol{a}_{0}^{k}, \mu_{k}\right) \\
& \cdot p\left(r_{k} \mid \boldsymbol{r}_{0}^{k-1}, \boldsymbol{a}_{0}^{k}, \mu_{k}\right) p\left(\boldsymbol{r}_{0}^{k-1} \mid \boldsymbol{a}_{0}^{k-1}, \mu_{k}\right) \\
& \cdot P\left\{\boldsymbol{a}_{0}^{k-1}, \mu_{k}\right\} P\left\{a_{k}\right\} .
\end{aligned}
$$

In general, the evaluation of the probability density functions (pdfs) in (2) requires the introduction of a tree diagram. However, the tree can be folded into a trellis if the conditional observation satisfies the following finite memory condition [28]:

$$
p\left(r_{k} \mid \boldsymbol{r}_{0}^{k-1}, \boldsymbol{a}_{0}^{k}, \mu_{k}\right)=p\left(r_{k} \mid \boldsymbol{r}_{0}^{k-1}, \boldsymbol{a}_{k-C}^{k}, \mu_{k}\right)
$$

where $C$ can be defined as a finite memory parameter. If this condition is exactly verified, an FB algorithm can be derived without any approximation [28]. The application of the finite memory condition leads naturally to the introduction of an augmented trellis diagram, where an augmented state $S_{k}$ is defined as $\left(\boldsymbol{a}_{k-C}^{k-1}, \mu_{k}\right)$, and the corresponding transition is $T_{k}=$ $\left(S_{k}, a_{k}\right)=\left(\boldsymbol{a}_{k-C}^{k}, \mu_{k}\right)=\left(\boldsymbol{a}_{k-C}^{k}, \mu_{k+1}\right)$. Obviously, there are $M^{C} \Omega$ augmented states.

If the finite memory condition is not verified in an exact sense, as in the case of channels with infinite memory, then it is not possible to fold the underlying tree structure implied by (2) into a trellis without approximation [30]. In this case, any solution leading to an FB algorithm is necessarily suboptimal. In particular, we consider the following approximation based on memory truncation:

$$
\begin{aligned}
p\left(r_{k} \mid \boldsymbol{r}_{0}^{k-1}, \boldsymbol{a}_{0}^{k}, \mu_{k}\right) & \simeq p\left(r_{k} \mid \boldsymbol{r}_{k-N}^{k-1}, \boldsymbol{a}_{k-N}^{k}, \mu_{k}\right) \\
& =p\left(r_{k} \mid \boldsymbol{r}_{k-N}^{k-1}, T_{k}\right)
\end{aligned}
$$

where $N$ represents a truncation depth and $T_{k}=\left(S_{k}, a_{k}\right)$, where $S_{k}$, for analogy with the case of strictly finite memory, is defined as $\left(\boldsymbol{a}_{k-N}^{k-1}, \mu_{k}\right)$. As a consequence of (4), the first pdf in the summation at the right-hand side of (2) can be approximated as

$$
p\left(\boldsymbol{r}_{k+1}^{K-1} \mid \boldsymbol{r}_{0}^{k}, \boldsymbol{a}_{0}^{k}, \mu_{k}\right) \simeq p\left(\boldsymbol{r}_{k+1}^{K-1} \mid \boldsymbol{r}_{k-N+1}^{k}, S_{k+1}\right) .
$$

Finally, based on (4) and (5), the exact APP in (2) can be approximated by the following reliability value:

$$
\begin{aligned}
S\left[a_{k}\right]= & \sum_{\boldsymbol{a}_{0}^{k-1}} \sum_{\mu_{k}} p\left(\boldsymbol{r}_{k+1}^{K-1} \mid \boldsymbol{r}_{k-N+1}^{k}, S_{k+1}\right) p\left(r_{k} \mid \boldsymbol{r}_{k-N}^{k-1}, T_{k}\right) \\
& \cdot p\left(\boldsymbol{r}_{0}^{k-1} \mid \boldsymbol{a}_{0}^{k-1}, \mu_{k}\right) P\left\{\boldsymbol{a}_{0}^{k-1}, \mu_{k}\right\} P\left\{a_{k}\right\} \\
= & \sum_{\boldsymbol{a}_{k-1}^{k-1}} \sum_{\mu_{k}} p\left(\boldsymbol{r}_{k+1}^{K-1} \mid \boldsymbol{r}_{k-N+1}^{k}, S_{k+1}\right) p\left(r_{k} \mid \boldsymbol{r}_{k-N}^{k-1}, T_{k}\right) \\
& \cdot\left[\sum_{\boldsymbol{a}_{0}^{k-N-1}} p\left(\boldsymbol{r}_{0}^{k-1} \mid \boldsymbol{a}_{0}^{k-1}, \mu_{k}\right) P\left\{\boldsymbol{a}_{0}^{k-1}, \mu_{k}\right\}\right] P\left\{a_{k}\right\} \\
= & \sum_{\boldsymbol{a}_{k-N}^{k-1} \sum_{\mu_{k}} \underbrace{p\left(\boldsymbol{r}_{k+1}^{K-1} \mid \boldsymbol{r}_{k-N+1}^{k}, S_{k+1}\right)}_{\beta_{k+1}\left(S_{k+1}\right)}} \\
& \cdot \underbrace{p\left(r_{k} \mid \boldsymbol{r}_{k-N}^{k-1}, T_{k}\right) P\left\{a_{k}\right\}}_{\gamma_{k}\left(T_{k}\right)}
\end{aligned}
$$

$$
\begin{aligned}
& \cdot \underbrace{p\left(\boldsymbol{r}_{0}^{k-1} \mid \boldsymbol{a}_{k-N}^{k-1}, \mu_{k}\right) P\left\{\boldsymbol{a}_{k-N}^{k-1}, \mu_{k}\right\}}_{\alpha_{k}\left(S_{k}\right)} \\
= & \sum_{T_{k}: a_{k}} \alpha_{k}\left(S_{k}\right) \gamma_{k}\left(T_{k}\right) \beta_{k+1}\left(S_{k+1}\right)
\end{aligned}
$$

where the notation $T_{k}: a_{k}$ indicates all transitions compatible with $a_{k}$. Note that the final expression (6) for the reliability value $S\left[a_{k}\right]$ holds for any type of encoder/modulator characterized by a regular trellis diagram. In particular, it holds for any type of encoder, either recursive or nonrecursive, provided that $S_{k}$ is redefined as $\left(\mu_{k-N}, \boldsymbol{a}_{k-N}^{k-1}\right)$, i.e., the encoder state to be considered in the augmented state $S_{k}$ is $\mu_{k-N}$, rather than the state $\mu_{k}$ considered in the initial definition. A rigorous and general derivation can be found in [28].

It is possible to show that the quantities $\alpha_{k}\left(S_{k}\right)$ and $\beta_{k+1}\left(S_{k+1}\right)$ can be calculated by means of the following forward and backward recursions [28]:

$$
\begin{aligned}
& \alpha_{k}\left(S_{k}\right)=\sum_{T_{k-1}: S_{k}} \alpha_{k-1}\left(S_{k-1}\right) \gamma_{k-1}\left(T_{k-1}\right) \\
& \beta_{k}\left(S_{k}\right)=\sum_{T_{k}: S_{k}} \beta_{k+1}\left(S_{k+1}\right) \gamma_{k}\left(T_{k}\right)
\end{aligned}
$$

where $\left\{\alpha_{0}\left(S_{0}\right)\right\}$ and $\left\{\beta_{K}\left(S_{K}\right)\right\}$ have to be properly initialized [28]. The basic quantity $\gamma_{k}\left(T_{k}\right)$, referred to as exponential ${ }^{4}$ metric, is used in both recursions (7), (8), and in the completion operation (6). This is why we refer to this class of algorithms as TM-FB algorithms with CR.

The class of TM-FB-CR algorithms represents a natural extension, to the case of transmission over channels with infinite memory, of the FB algorithm (optimal for detection over channels with strictly finite memory [31]). Fig. 2 depicts the basic operations in a TM-FB-CR algorithm.

- In Fig. 2(a), a pictorial description of the step at epoch $k$ in the forward recursion is shown. In particular, the exponential metric $\gamma_{k-1}$, evaluated in correspondence with each transition $T_{k-1}$, is used to compute $\left\{\alpha_{k}\left(S_{k}\right)\right\}$ from $\left\{\alpha_{k-1}\left(S_{k-1}\right)\right\}$. In the figure, it is highlighted that in the evaluation of $\gamma_{k-1}$, the observation at epoch $k-1$, i.e., $r_{k-1}$, is conditioned on the $N$ previous observations $\boldsymbol{r}_{k-N-1}^{k-2}$.

- In Fig. 2(b), a pictorial description of the step at epoch $k+1$ in the backward recursion is shown. In particular, the exponential metric $\gamma_{k+1}$, evaluated in correspondence with each transition $T_{k+1}$, is used to compute $\left\{\beta_{k+1}\left(S_{k+1}\right)\right\}$ from $\left\{\beta_{k+2}\left(S_{k+2}\right)\right\}$.

- Finally, in Fig. 2(c), a pictorial description of the completion at epoch $k$ is shown. The exponential metric $\gamma_{k}$, evaluated in correspondence with each transition $T_{k}$, is used to combine $\left\{\alpha_{k}\left(S_{k}\right)\right\}$ with $\left\{\beta_{k+1}\left(S_{k+1}\right)\right\}$.

We remark that TM-FB-CR algorithms are suitable for the application of the state-reduction technique proposed in [24], where a reduced state is obtained by substituting $N$ by a $r e-$ duced-state parameter $Q<N$. In particular, forward per-state decision-feedback (DF) sequences are constructed during the

\footnotetext{
${ }^{4}$ The usual notation in the literature refers to a metric in the logarithmic domain. Hence, assuming that $\log \gamma_{k}$ can be referred to as metric, we refer to $\gamma_{k}$ as an exponential metric.
} 


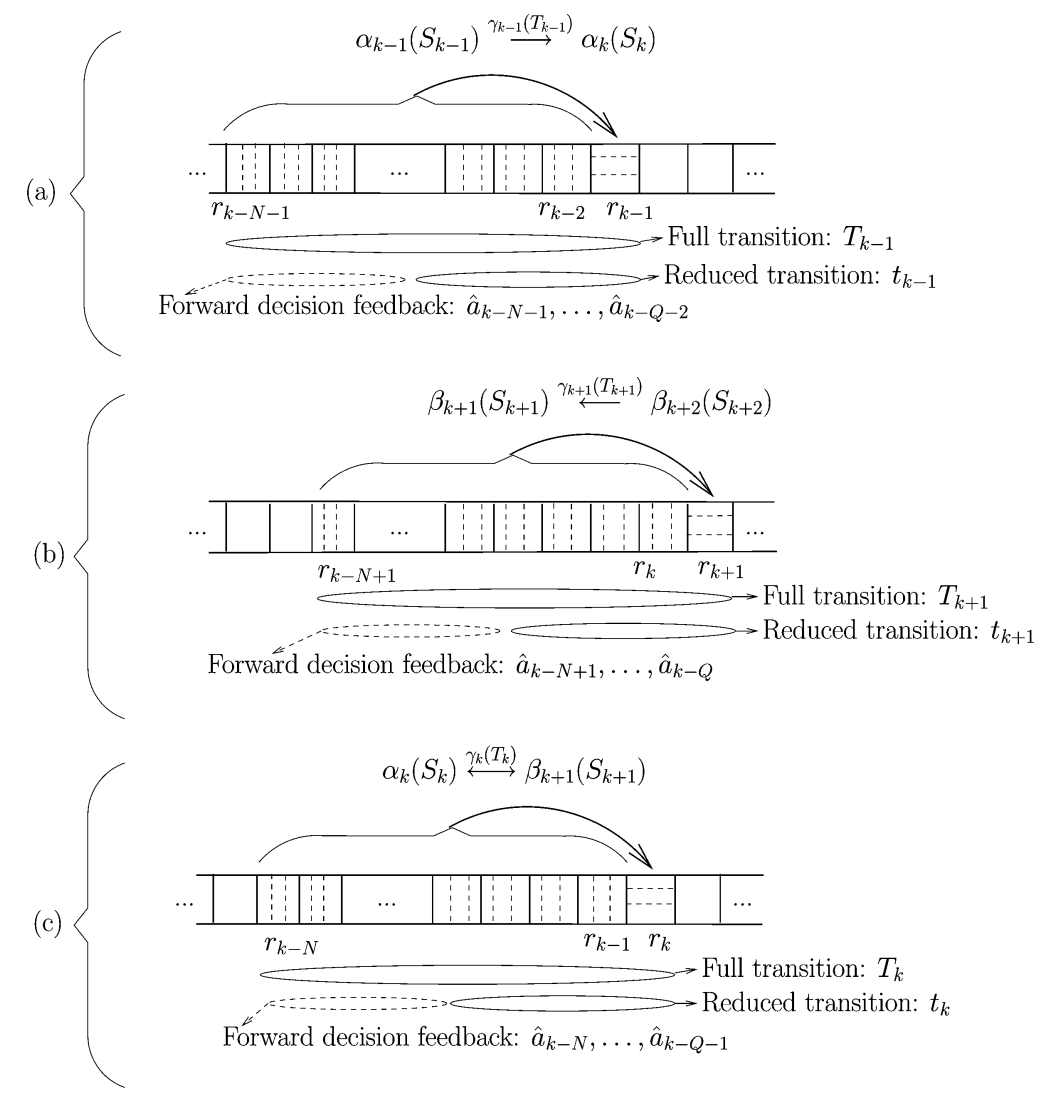

Fig. 2. Step at the $k$ th epoch for a TM-FB-CR algorithm. (a) Forward recursion. (b) Backward recursion. (c) Completion.

forward recursion, and used in the backward recursion and completion. In Fig. 2, the reduced transition, together with the indicated forward DF sequence of information symbols, is depicted for (a) a forward recursion step, (b) a backward recursion step, and (c) a completion step. The complexity of TM-FB-CR algorithms and their reduced-state versions will be discussed in detail in Section IV.

2) TM-FB-DR Algorithms: The class of TM-FB-CR algorithms has been derived from a specific approach to the computation of the APP given in (1). In the following, we propose an alternative approach, such that the forward and backward recursions are decoupled. In fact, the APP in (1) can be manipulated as follows:

$$
\begin{aligned}
P\left\{a_{k} \mid \boldsymbol{r}\right\} \propto & \sum_{\boldsymbol{a}_{0}^{k-1}} \sum_{\mu_{k}} \sum_{\boldsymbol{a}_{k+1}^{K-1}} p\left(\boldsymbol{r} \mid \boldsymbol{a}, \mu_{k}\right) P\left\{\boldsymbol{a}, \mu_{k}\right\} \\
= & \sum_{\boldsymbol{a}_{0}^{k-1}} \sum_{\mu_{k}} \sum_{\boldsymbol{a}_{k+1}^{K-1}} p\left(\boldsymbol{r}_{k-L}^{k+L} \mid \boldsymbol{r}_{0}^{k-L-1}, \boldsymbol{r}_{k+L+1}^{K-1}, \boldsymbol{a}, \mu_{k}\right) \\
& \cdot p\left(\boldsymbol{r}_{k+L+1}^{K-1} \mid \boldsymbol{r}_{0}^{k-L-1}, \boldsymbol{a}, \mu_{k}\right) \\
& \cdot P\left\{\boldsymbol{a}_{k-L}^{K-1} \mid \boldsymbol{r}_{0}^{k-L-1}, \boldsymbol{a}_{0}^{k-L-1}, \mu_{k-L}\right\} \\
& \cdot p\left(\boldsymbol{r}_{0}^{k-L-1} \mid \boldsymbol{a}_{0}^{k-L-1}, \mu_{k-L}\right) P\left\{\boldsymbol{a}_{0}^{k-L-1}, \mu_{k-L}\right\} \\
= & \sum_{\boldsymbol{a}_{0}^{k-1}} \sum_{\mu_{k}} \sum_{\boldsymbol{a}_{k+1}^{K-1}} p\left(\boldsymbol{r}_{k-L}^{k+L} \mid \boldsymbol{r}_{0}^{k-L-1}, \boldsymbol{r}_{k+L+1}^{K-1}, \boldsymbol{a}, \mu_{k}\right) \\
& \cdot p\left(\boldsymbol{r}_{k+1}^{K-1} \mid \boldsymbol{r}_{0}^{k-L-1}, \boldsymbol{a}, \mu_{k}\right) \\
& \cdot p\left(\boldsymbol{r}_{0}^{k-L-1} \mid \boldsymbol{a}_{0}^{k-L-1}, \mu_{k-L}\right) P\left\{\boldsymbol{a}_{0}^{k-L-1}, \mu_{k-L}\right\} \\
& \cdot P\left\{\boldsymbol{a}_{k-L}^{K-1}\right\}
\end{aligned}
$$

where $L$ will be referred to as completion parameter. In this case, memory truncation can be applied, obtaining

$$
\begin{aligned}
& p\left(\boldsymbol{r}_{k-L}^{k+L} \mid \boldsymbol{r}_{0}^{k-L-1}, \boldsymbol{r}_{k+L+1}^{K-1}, \boldsymbol{a}, \mu_{k}\right) \\
& \quad \simeq p\left(\boldsymbol{r}_{k-L}^{k+L} \mid \boldsymbol{r}_{k-L-N}^{k-L-1}, \boldsymbol{r}_{k+L+1}^{k+L+N}, \boldsymbol{a}_{k-L-N}^{k+L+N}, \mu_{k}\right) .
\end{aligned}
$$

Based on (10), we can further approximate (9) as follows:

$$
\begin{aligned}
& P\left\{a_{k} \mid \boldsymbol{r}\right\} \\
& \simeq \sum_{\boldsymbol{a}_{0}^{k-1}} \sum_{\mu_{k}} \sum_{\boldsymbol{a}_{k+1}^{K-1}} p\left(\boldsymbol{r}_{k-L}^{k+L} \mid \boldsymbol{r}_{k-L-N}^{k-L-1}, \boldsymbol{r}_{k+L+1}^{k+L+N}, \boldsymbol{a}_{k-L-N}^{k+L+N}, \mu_{k}\right) \\
& \quad \cdot p\left(\boldsymbol{r}_{k+L+1}^{K-1} \mid \boldsymbol{r}_{0}^{k-L-1}, \boldsymbol{a}, \mu_{k}\right) p\left(\boldsymbol{r}_{0}^{k-L-1} \mid \boldsymbol{a}_{0}^{k-L-1}, \mu_{k-L}\right) \\
& \quad \cdot P\left\{\boldsymbol{a}_{0}^{k-L-1}, \mu_{k-L}\right\} P\left\{\boldsymbol{a}_{k-L}^{K-1}\right\} .
\end{aligned}
$$

If we assume $2 L \geq N$, memory truncation allows one to write

$$
p\left(\boldsymbol{r}_{k+L+1}^{K-1} \mid \boldsymbol{r}_{0}^{k-L-1}, \boldsymbol{a}, \mu_{k}\right) \simeq p\left(\boldsymbol{r}_{k+L+1}^{K-1} \mid \boldsymbol{a}_{k+L+1}^{K-1}, \mu_{k+L+1}\right) .
$$

Using (12) in (11) and recalling the independence between information symbols, it is possible to obtain the reliability value (which approximates the APP) shown in (13) at the bottom of the next page. The quantities $A_{k}\left(S_{k}\right)$ and $B_{k}\left(S_{k+N}\right)$ can be computed by means of forward and backward recursions, respectively [32]

$$
\begin{aligned}
A_{k}\left(S_{k}\right) & =\sum_{T_{k-1}: S_{k}} A_{k-1}\left(S_{k-1}\right) G_{k-1}^{F}\left(T_{k-1}\right) \\
B_{k}\left(S_{k+N}\right) & =\sum_{T_{k+N}: S_{k+N}} B_{k+1}\left(S_{k+N+1}\right) G_{k}^{B}\left(T_{k+N}\right)
\end{aligned}
$$


where

$$
\begin{aligned}
G_{k-1}^{F}\left(T_{k-1}\right) & \triangleq \gamma_{k-1}\left(T_{k-1}\right) \\
& =p\left(r_{k-1} \mid \boldsymbol{r}_{k-N-1}^{k-2}, T_{k-1}\right) P\left\{a_{k-1}\right\} \\
G_{k}^{B}\left(T_{k+N}\right) & \triangleq p\left(r_{k} \mid \boldsymbol{r}_{k+1}^{k+N}, T_{k+N}\right) P\left\{a_{k+N}\right\} .
\end{aligned}
$$

Provided that $A_{0}\left(S_{0}\right)=\alpha_{0}\left(S_{0}\right) \forall S_{0}$, one can conclude that $A_{k}\left(S_{k}\right)=\alpha_{k}\left(S_{k}\right) \forall S_{k}$, since in the forward recursion of a TM-FB-DR algorithm, the same exponential metric as in the forward recursion of a TM-FB-CR algorithm is used.

Use of memory truncation in (10) leads to the presence, in the completion at the $k$ th epoch, of a symmetric window of observations centered around the sequence $\boldsymbol{r}_{k-L}^{k+L}$. A simple drawing highlighting the basic operations of TM-FB-DR algorithms is shown in Fig. 3.

- In Fig. 3(a), a pictorial description of the step at epoch $k$ in the forward recursion is shown. The exponential metric $G_{k-L-1}^{F}\left(T_{k-L-1}\right)$, evaluated in correspondence with each transition $T_{k-L-1}$, is used to compute $\left\{A_{k-L}\left(S_{k-L}\right)\right\}$ from $\left\{A_{k-L-1}\left(S_{k-L-1}\right)\right\}$. In the figure, it is highlighted that in the evaluation of $G_{k-1}^{F}\left(T_{k-1}\right)$, the observation at epoch $k-L-1$, i.e., $r_{k-L-1}$, is conditioned on the $N$ past observations $\boldsymbol{r}_{k-L-N-1}^{k-L-2}$.

- In Fig. 3(b), a pictorial description of the step at epoch $k+1$ in the backward recursion is shown. The exponential metric $G_{k+L+1}^{B}\left(T_{k+L+N+1}\right)$, evaluated in correspondence to each transition $T_{k+L+N+1}$, is used to compute $\left\{B_{k+L+1}\left(S_{k+L+N+1}\right)\right\}$ from $\left\{B_{k+L+2}\left(S_{k+L+N+2}\right)\right\}$. In the computation of $G_{k+L+1}^{B}\left(T_{k+L+N+1}\right)$, the observation at epoch $k+L+1$ is conditioned on the $N$ future observations $\boldsymbol{r}_{k+L+2}^{k+L+N+1}$.
- Finally, in Fig. 3(c), a pictorial description of the completion at epoch $k$ is shown. The exponential metric $G_{k}^{C}\left(S_{k-L}, \boldsymbol{a}_{k-L}^{k+L+N}\right)$, evaluated in correspondence with a group of multiple transitions $\left(T_{k-L}, \ldots, T_{k+L+N}\right)$, is used to combine $\left\{A_{k-L}\left(S_{k-L}\right)\right\}$ with $\left\{B_{k+L+1}\left(S_{k+L+N+1}\right)\right\}$. Note that in the computation of $G_{k}^{C}\left(S_{k-L}, \boldsymbol{a}_{k-L}^{k+L+N}\right)$, the $2 L+1$ observations $\boldsymbol{r}_{k-L}^{k+L}$ are conditioned on $N$ "past" observations $\boldsymbol{r}_{k-L-N}^{k-L-1}$ and $N$ "future" observations $\boldsymbol{r}_{k+L+1}^{k+L+N}$. One can observe that while in a TM-FB-CR algorithm, exactly the same metric is used in both recursions, in a TM-FB-DR algorithm, the metric used in the backward recursion is the mirror image version of that used in the forward recursion. In other words, the conditioning observations in the forward recursion are in the past, whereas in the backward recursion, they are in the future. In this sense, TM-FB-DR algorithms can not be considered as a natural extension of the FB algorithm, since the memory-truncation assumption is applied specularly (and not identically) in the two recursions. This leads to the presence, in TM-FB-DR algorithms, of a completion operation based on an extended window of observations. This type of completion will simply be referred to as extended.

The complexity of this class of TM-FB algorithms will be reduced by applying the generalized state-reduction techniques for FB algorithms proposed in [25]. In particular, the parameter $N$ is replaced by a reduced-state parameter $Q$, and the condition $2 L \geq N$, which leads to the decoupling condition given by (12), reduces to $2 L \geq Q$. In Fig. 3, in each recursion, the reduced transition is indicated, together with the indicated DF sequence of information symbols. Unlike in a reduced-state TM-FB-CR algorithm, in this case, a forward DF is built during the forward recursion, whereas a backward DF, independent of the forward $\mathrm{DF}$, is built during the backward recursion. In the completion,

$$
\begin{aligned}
& S\left[a_{k}\right]=\sum_{\boldsymbol{a}_{0}^{k-1}} \sum_{\mu_{k}} \sum_{\boldsymbol{a}_{k+1}^{K-1}} p\left(\boldsymbol{r}_{k-L}^{k+L} \mid \boldsymbol{r}_{k-L-N}^{k-L-1}, \boldsymbol{r}_{k+L+1}^{k+L+N}, \boldsymbol{a}_{k-L-N}^{k+L+N}, \mu_{k}\right) \\
& \text {-p }\left(\boldsymbol{r}_{k+L+1}^{K-1} \mid \boldsymbol{a}_{k+L+1}^{K-1}, \mu_{k+L+1}\right) p\left(\boldsymbol{r}_{0}^{k-L-1}, \boldsymbol{a}_{0}^{k-L-1}, \mu_{k-L}\right) P\left\{\boldsymbol{a}_{k-L}^{K-1}\right\} \\
& =\sum_{\boldsymbol{a}_{k-N-L}^{k-1}} \sum_{\mu_{k}} \sum_{\boldsymbol{a}_{k+1}^{k+N+L}} p\left(\boldsymbol{r}_{k-L}^{k+L} \mid \boldsymbol{r}_{k-L-N}^{k-L-1}, \boldsymbol{r}_{k+L+1}^{k+L+N}, \boldsymbol{a}_{k-L-N}^{k+L+N}, \mu_{k}\right) \\
& \text { - }\left[\sum_{\boldsymbol{a}_{k+N+L+1}^{K-1}} p\left(\boldsymbol{r}_{k+L+1}^{K-1} \mid \boldsymbol{a}_{k+L+1}^{K-1}, \mu_{k+L+1}\right) P\left\{\boldsymbol{a}_{k+N+L+1}^{K-1}\right\}\right] \\
& \cdot\left[\sum_{\boldsymbol{a}_{0}^{k-N-L-1}} p\left(\boldsymbol{r}_{0}^{k-L-1} \mid \boldsymbol{a}_{0}^{k-L-1}, \mu_{k-L}\right) P\left\{\boldsymbol{a}_{0}^{k-L-1}, \mu_{k-L}\right\}\right] P\left\{\boldsymbol{a}_{k-L}^{k+L+N}\right\} \\
& =\sum_{\boldsymbol{a}_{k-N-L}^{k-1}} \sum_{\mu_{k}} \sum_{\boldsymbol{a}_{k+1}^{k+N+L}} \underbrace{p\left(\boldsymbol{r}_{k-L}^{k+L} \mid \boldsymbol{r}_{k-L-N}^{k-L-1}, \boldsymbol{r}_{k+L+1}^{k+L+N}, \boldsymbol{a}_{k-L-N}^{k+L+N}, \mu_{k}\right) P\left\{\boldsymbol{a}_{k-L}^{k+L+N}\right\}}_{G_{k}^{C}\left(S_{k-L}, \boldsymbol{a}_{k-L}^{k+L+N}\right)} \\
& \cdot \underbrace{p\left(\boldsymbol{r}_{k+L+1}^{K-1} \mid \boldsymbol{a}_{k+L+1}^{k+L+N}, \mu_{k+L+1}\right)}_{B_{k+L+1}\left(S_{k+L+N+1}\right)} \underbrace{p\left(\boldsymbol{r}_{0}^{k-L-1} \mid \boldsymbol{a}_{k-L-N}^{k-L-1}, \mu_{k-L}\right) P\left\{\boldsymbol{a}_{k-L-N}^{k-L-1}, \mu_{k-L}\right\}}_{A_{k-L}\left(S_{k-L}\right)} \\
& =\sum_{S_{k-L}} \sum_{\boldsymbol{a}_{k-L}^{k+L+N-1}: a_{k}} A_{k-L}\left(S_{k-L}\right) G_{k}^{C}\left(S_{k-L}, \boldsymbol{a}_{k-L}^{k+L+N}\right) B_{k+L+1}\left(S_{k+L+N+1}\right)
\end{aligned}
$$


(a)

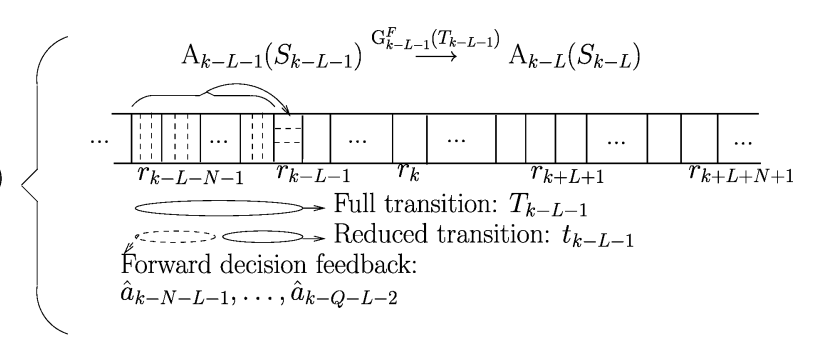

(b)
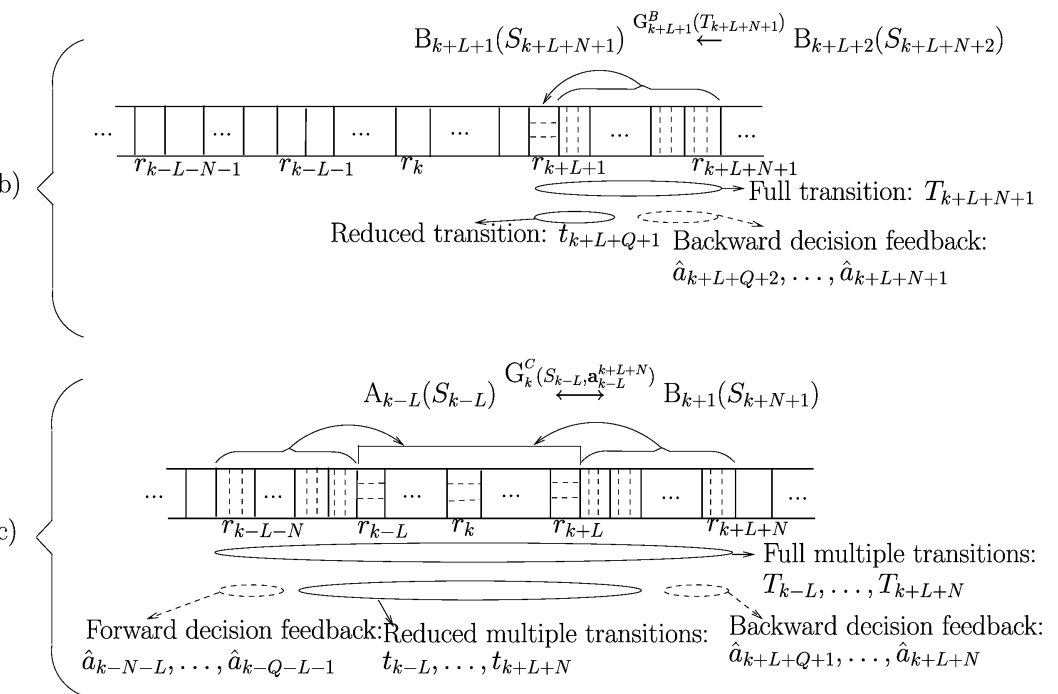

Fig. 3. Step at the $k$ th epoch for a TM-FB-DR algorithm. (a) Forward recursion (a). (b) Backward recursion. (c) Completion.

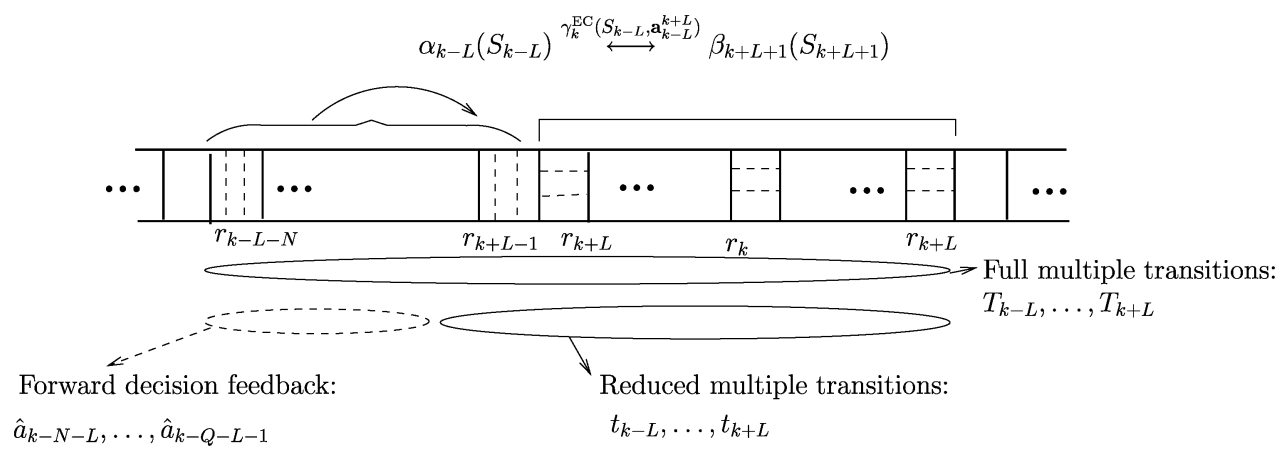

Fig. 4. Extended completion at the $k$ th epoch for a TM-FB-CR algorithm.

the multiple transitions are "compressed" toward the center, and forward and backward DF sequences, built during the two recursions, are used at the "extremities" of the original multiple transitions' group. A complexity analysis of this class of TM-FB algorithms and their reduced-state versions will be considered in Section IV.

3) TM-FB-CR Algorithms With Extended Completion: We have shown that TM-FB-DR algorithms differ from TM-FB-CR algorithms in two main aspects: 1) the two recursions are decoupled; and 2) there is an extended completion. Motivated by this observation, it is interesting to generalize the proposed class of TM-FB-CR algorithms in order to incorporate an extended completion, where multiple transitions are considered. In particular, the generated soft-output value is computed as follows:

$$
\begin{array}{r}
S\left[a_{k}\right]=\sum_{S_{k-L}} \sum_{\boldsymbol{a}_{k-L}^{k+L}: a_{k}} \alpha_{k-L}\left(S_{k-L}\right) \gamma_{k}^{\mathrm{EC}} \cdot\left(S_{k-L},\right. \\
\left.\boldsymbol{a}_{k-L}^{k+L}\right) \beta_{k+L+1}\left(S_{k+L+1}\right)
\end{array}
$$

where $a_{k-L}^{k+L}: a_{k}$ denotes all information sequences compatible with $a_{k}$, and

$$
\begin{array}{r}
\gamma_{k}^{\mathrm{EC}}\left(S_{k-L}, \boldsymbol{a}_{k-L}^{k+L}\right) \triangleq p\left(\boldsymbol{r}_{k-L}^{k+L} \mid \boldsymbol{r}_{k-L-N}^{k-L-1}, S_{k-L}, \boldsymbol{a}_{k-L}^{k+L}\right) \\
\cdot P\left\{\boldsymbol{a}_{k-L}^{k+L}\right\}
\end{array}
$$

The extended completion operation for generalized TM-FB-CR algorithms is shown in Fig. 4. Unlike in the completion of the previously derived TM-FB-CR algorithms without extended completion, for these generalized TM-FB-CR algorithms, the exponential metric used at the completion operation is such that the group of observations $\boldsymbol{r}_{k-L}^{k+L}$, rather than the single observation $r_{k}$, is conditioned on $N$ previous observations.

One can observe that the design of TM-FB-CR algorithms with extended completion is dictated by intuition, and it does not derive as a direct approximated evaluation of the APP, through the application of the chain rule factorization. However, since approximations are involved, the performance offered by this generalized class of TM-FB-CR algorithms is worth evaluating. 
Since the case with $L=0$ corresponds to the original class of TM-FB-CR algorithms, in the following, we will simply refer to TM-FB-CR algorithms as characterized by a general value of the completion parameter $L$.

\section{B. TM Sequence Detection: Viterbi Algorithm}

The starting point is the computation of the MAP sequence

$$
\hat{\boldsymbol{a}}=\arg \max _{\boldsymbol{a}} p(\boldsymbol{r} \mid \boldsymbol{a}) P\{\boldsymbol{a}\} .
$$

An efficient way to recursively determine the sequence of estimated information symbols is based on a VA [33]. We now clearly show the relation between TM-VA and TM-FB algorithms.

By applying the max-log [34] or min-sum [35] approximation and defining path and branch metrics as $\bar{\alpha}_{k} \triangleq \log \alpha_{k}$ and $\bar{\gamma}_{k} \triangleq \log \gamma_{k}$, respectively, the forward recursion (7) can be approximated as follows:

$$
\begin{aligned}
\bar{\alpha}_{k}\left(S_{k}\right) & =\log \sum_{T_{k-1}: S_{k}} \exp \left[\bar{\alpha}_{k-1}\left(S_{k-1}\right)+\bar{\gamma}_{k-1}\left(T_{k-1}\right)\right] \\
& \simeq \max _{T_{k-1}: S_{k}}\left[\bar{\alpha}_{k-1}\left(S_{k-1}\right)+\bar{\gamma}_{k-1}\left(T_{k-1}\right)\right]
\end{aligned}
$$

This relationship corresponds to the recursive computation of a VA, which can thus be referred to as TM-VA. In fact, all the proposed approaches to the design of a VA appeared in the literature, and based on memory truncation, reduce to a recursive formulation which can be generally written as follows (provided that state $S_{k}$ and transition $T_{k}$ are defined as in (21)):

$$
m_{k}\left(S_{k}\right)=\max _{T_{k-1}: S_{k}}\left[m_{k-1}\left(S_{k-1}\right)+\lambda_{k-1}\left(T_{k-1}\right)\right]
$$

where $m_{k}\left(S_{k}\right)$ and $\lambda_{k-1}$ represents path and branch metrics, respectively, and

$$
\max _{S_{K}} m_{K}\left(S_{K}\right) \simeq \max _{\boldsymbol{a}}[p(\boldsymbol{r} \mid \boldsymbol{a}) P\{\boldsymbol{a}\}]
$$

The recursive maximization (22) [culminating with (23)] can be implemented by means of a trellis search based on a VA. By identifying $\bar{\alpha}_{k}=m_{k}$ and $\bar{\gamma}_{k}=\lambda_{k}$, it is immediately recognizable that a TM-FB-CR algorithm is strictly related to a TM-VA. Note that the max-log approximation can also be applied to TM-FB-DR algorithms - the extension of the proposed formulation is straightforward, by substituting multiplications with additions, and additions with maximizations.

To conclude this brief reinterpretation of a TM-VA, we point out that the idea of extended completion, introduced for TM-FB algorithms, could be applied to a TM-VA, for example, to improve the survivor selection. This extension, however, is beyond the scope of this paper.

\section{COMPleXITy CONSIDERATIONS}

Without losing generality, we derive a simple, although insightful, analysis of the complexity of TM-FB algorithms. In particular, we assume that the max-log approximation is used. The complexity per trellis section of a TM-VA is roughly comparable to that of a recursion in a TM-FB algorithm. We first analyze the complexity of TM-FB-CR and TM-FB-DR algorithms in the absence of state reduction, and then extend this analysis to the case where state-reduction techniques are applied. Our complexity analysis does not account for the complexity of the exponential metrics computation.

\section{A. Absence of State Reduction}

We first consider the class of TM-FB-CR algorithms (possibly with extended completion).

- Considering the forward recursion and taking into account the application of the max-log approximation, it follows that at each epoch and state (there are $M^{N} \Omega$ states), $M$ additions have to be performed (in correspondence to the incoming branches), followed by a comparison among the generated values. Hence, a total of $M^{N} \Omega$ additions and $M^{N+1} \Omega$ comparisons are required in each step of the forward recursion.

- The backward recursion has, obviously, the same complexity of the forward recursion.

- In the completion, in order to generate the soft-output reliability value relative to a particular symbol value at a given epoch (out of the $M$ possible ones), all the multiple transitions associated with this symbol have to be considered. There are $M^{N+2 L+1} \Omega$ such transitions, and an addition must be carried out in correspondence to each of them. Hence, a comparison among these values has to be performed to choose the maximum. Since these operations are required for each possible symbol value, a total of $M^{N+2 L+1} \Omega M$ additions and $M$ comparisons are required in the completion operation at any epoch.

We now turn our attention to the case of TM-FB-DR algorithms.

- Since the forward recursion is the same as that of TM-FB-CR algorithms, the complexity is obviously the same.

- The backward recursion uses a metric which is the mirrorimage version of that used in the forward recursion, and this does not affect complexity. Hence, the complexity is the same as in the forward recursion.

Note, however, that if the metrics $\left\{\gamma_{k}\right\}$ computed in the forward recursion of TM-FB-CR algorithms are stored for later use, then the backward recursion of TM-FB-CR algorithms might be significantly less complex than the backward recursion of TM-FB-DR algorithms. Obviously, the potential complexity reduction of the backward recursion of TM-FB-DR algorithms comes at the cost of a significant amount of memory occupation. The complexity analysis proposed in this paper can be extended in order to take into account possible tradeoffs between computational complexity and memory occupation.

- At the completion, in order to generate a reliability value for each possible symbol value at a given epoch, $M^{N} \Omega M^{2 L+N+1}$ additions have to be considered, and the maximum of the generated quantities has to be determined through a comparison operation. Since there are $M$ reliability values to be generated, it follows that the overall numbers of additions and comparisons per trellis section are $M^{N} \Omega M^{2 L+N+1} M$ and $M$, respectively. 
TABLE I

Complexity Per Trellis Section, in Terms of Additions and Comparisons, of Full-State TM-FB Algorithms With MaX-Log ApProXimation

\begin{tabular}{c|c|c|c|c|c|c}
\hline & \multicolumn{2}{|l}{ Forward and Backward Recursions } & \multicolumn{2}{c|}{ Completion } & \multicolumn{2}{c}{ Total } \\
\hline & Add. & Comp. & Add. & Comp. & Add. & Comp. \\
\hline \hline TM-FB-CR & $M^{N+1} \Omega$ & $M^{N} \Omega$ & $M^{2 L+N+2} \Omega$ & $M$ & $\Omega M^{N+1}\left(M^{2 L+1}+2\right)$ & $2 M^{N} \Omega+M^{2 L}$ \\
\hline TM-FB-DR & $M^{N+1} \Omega$ & $M^{N} \Omega$ & $M^{2(N+L+1)} \Omega$ & $M$ & $\Omega M^{N+1}\left(2+M^{N+2 L+1}\right)$ & $2 M^{N} \Omega+M^{2 L}$ \\
\hline
\end{tabular}

TABLE II

Complexity Per Trellis Section, in Terms of Memory Access, of Reduced-State TM-FB Algorithms With MaX-Log Approximation

\begin{tabular}{l|c|c|c}
\hline & Forward and Backward Recursions & Completion & Total \\
\hline & Mem. Acc. & Mem. Acc. & Mem. Acc. \\
\hline \hline TM-FB-CR & $M^{Q+1} \Omega$ & $\Omega M^{Q+2 L+2}$ & $\Omega M^{Q+1}\left(2+M^{2 L+1}\right)$ \\
\hline TM-FB-DR & $M^{Q+1} \Omega$ & $2 \Omega M^{2(Q+L+1)}$ & $\Omega M^{Q+1} 2\left(1+M^{Q+2 L+1}\right)$ \\
\hline
\end{tabular}

A summary of the complexity levels of the proposed TM-FB algorithms, in terms of additions and comparisons per trellis section, is shown in Table I. We denote the total number of additions and comparisons as $\zeta_{a}(N, L)$ and $\zeta_{c}(N, L)$, respectively. From Table I, it is immediate to see that while $\zeta_{c}^{\mathrm{TM}-\mathrm{FB}-\mathrm{DR}}(N, L)=$ $\zeta_{c}^{\mathrm{TM}-\mathrm{FB}-\mathrm{DR}}(N, L)$, it holds that

$$
\begin{aligned}
\frac{\zeta_{a}^{\mathrm{TM}-\mathrm{FB}-\mathrm{DR}}(N, L)}{\zeta_{a}^{\mathrm{TM}-\mathrm{FB}-\mathrm{DR}}(N, L)} & =\frac{\Omega M^{N+1}\left(2+M^{2 L+1}\right)}{\Omega M^{N+1}\left(2+M^{N+2 L+1}\right)} \\
& =\frac{2+M^{2 L+1}}{2+M^{N+2 L+1}}<1 .
\end{aligned}
$$

Hence, TM-FB-DR algorithms are more complex, for any given values of $N$ and $L$, than TM-FB-CR algorithms. Note, however, that the recursions have the same complexity in both algorithmic classes, so that the increased complexity of TM-FB-DR algorithms is concentrated only in the completion operation.

\section{B. Presence of State Reduction}

As previously mentioned, in the case of state reduction, the formulation of TM-FB algorithms proposed in Section III can be straightforwardly extended by replacing $N$ with $Q$ in the two recursions. In particular, the results shown in Table I can be applied by substituting $N$ with $Q$. One can conclude that in terms of comparisons, it holds that $\zeta_{c}^{\mathrm{TM}-\mathrm{FB}-\mathrm{DR}}(Q, L)=$ $\zeta_{c}^{\mathrm{TM}-\mathrm{FB}-\mathrm{DR}}(Q, L)$, whereas in terms of additions, one can write

$$
\frac{\zeta_{a}^{\mathrm{TM}-\mathrm{FB}-\mathrm{DR}}(Q, L)}{\zeta_{a}^{\mathrm{TM}-\mathrm{FB}-\mathrm{DR}}(Q, L)}=\frac{2+M^{2 L+1}}{2+M^{Q+2 L+1}} .
$$

In the case of state reduction, DF has to be used. In order to perform numerical simulations, we associate with each reduced state the corresponding full state, based on suitably decided symbols. Hence, in correspondence to each reduced state, the associated full state must be accessed, and we refer to this basic operation as memory access. We now outline a reasonable analysis of the complexity of the proposed algorithmic classes in terms of memory access.

- In the case of TM-FB-CR algorithms, the same basic exponential metric $\gamma_{k}$ is used in both recursions. The computation of $\gamma_{k}$ for each trellis transition requires one memory access (the full state associated with the starting state of the reduced transition must be retrieved). In the central completion, the computation of $\gamma_{k}^{\mathrm{EC}}$ requires a single memory access, as well. Since for each addition, the metric $\gamma_{k}$ ( or $\gamma_{k}^{\mathrm{EC}}$ ) has to be computed, the complexity, in terms of memory access, is simply given by the complexity in terms of additions.

- In the case of TM-FB-DR algorithms, the same considerations as for TM-FB-CR algorithms hold in the two recursions. However, in the completion operation, in order to compute the extended metric $G_{k}^{C}$, two memory accesses are required (to the forward DF and to the backward DF associated with the starting and ending reduced states of the multiple transitions window, respectively).

A summary of the complexity per trellis section, in terms of memory access to stored symbol decisions, is shown in Table II. Defining the overall complexities, in terms of memory access, of TM-FB-CR and TM-FB-DR algorithms as $\zeta_{m}^{\text {TM-FB-DR }}(Q, L)$ and $\zeta_{m}^{\mathrm{TM}-\mathrm{FB}-\mathrm{DR}}(Q, L)$, respectively, it is immediate to conclude that

$$
\begin{aligned}
\frac{\zeta_{m}^{\mathrm{TM}-\mathrm{FB}-\mathrm{DR}}(Q, L)}{\zeta_{m}^{\mathrm{TM}-\mathrm{FB}-\mathrm{CR}}(Q, L)} & =\frac{\Omega M^{Q+1}\left(2+M^{2 L+1}\right)}{\Omega M^{Q+1} 2\left(1+M^{Q+2 L+1}\right)} \\
& =\frac{2+M^{2 L+1}}{2\left(1+M^{Q+2 L+1}\right)}<1 .
\end{aligned}
$$

As for additions and comparisons, the increased complexity, in terms of memory access, of TM-FB-DR algorithms is concentrated in the completion.

\section{Application to Stochastic ChanNELS}

A general model for the observation $r_{k}$ at the output of a stochastic channel is the following:

$$
r_{k}=f\left(\mu_{k}, a_{k}, \boldsymbol{\theta}_{k}\right)+n_{k}
$$

where $f(\cdot, \cdot, \cdot)$ is a known deterministic function, $\boldsymbol{\theta}_{k}$ represents a stochastic (vector) channel parameter at the $k$ th epoch and $\left\{n_{k}\right\}$ is a complex AWGN process, with variance $N_{0}$. In this case, the following two detection approaches can be devised from the general TM detection framework proposed in Section III.

1) The exponential metric $\gamma_{k}$ used in each recursion of TM-FB-CR algorithms (and in the forward recursion 
of TM-FB-DR algorithms) can be exactly computed as follows:

$\gamma_{k}\left(T_{k}\right)=\frac{E_{\boldsymbol{\theta}_{k-N}^{k}}\left\{p\left(\boldsymbol{r}_{k-N}^{k} \mid \boldsymbol{\theta}_{k-N}^{k}, T_{k}\right)\right\}}{E_{\boldsymbol{\theta}_{k-N}^{k-1}}\left\{p\left(\boldsymbol{r}_{k-N}^{k-1} \mid \boldsymbol{\theta}_{k-N}^{k-1}, S_{k}\right)\right\}} P\left\{a_{k}\right\}$

where the symbol $E_{*}\{\cdot\}$ denotes expectation with respect to $*$. Note that the average pdfs at the numerator and denominator differ only in the number of observables. Similarly, the exponential metric $\gamma_{k}^{\mathrm{EC}}$ used in TM-FB-CR algorithms with extended completion, and the exponential metrics $G_{k}^{F}, G_{k}^{B}$, and $G_{k}^{C}$ used in TM-FB-DR algorithms can be expressed as ratios of two average pdfs using a suitable number of observables. Hence, the derivation of TM-FB algorithms is simply based on the computation of an average pdf of a sequence of observations of arbitrary length. Specific metric expressions depend on the statistical description of the channel parameters $\left\{\boldsymbol{\theta}_{k}\right\}$.

2) An alternative approach consists of estimating, on the basis of a suitable observation window, the unknown parameter. Indicating by $\hat{\boldsymbol{\theta}}_{k}$ an estimate of the channel parameter, the exponential metric $\gamma_{k}$ can be written as follows:

$\gamma_{k}\left(T_{k}\right) \propto \exp \left\{\frac{\left|r_{k}-f\left(\mu_{k}, a_{k}, \hat{\boldsymbol{\theta}}_{k}\right)\right|^{2}}{N_{0}}\right\} P\left\{a_{k}\right\}$

where $\hat{\boldsymbol{\theta}}_{k}=\hat{\boldsymbol{\theta}}_{k}\left(S_{k}, \boldsymbol{r}_{k-N}^{k-1}\right)$. The specific estimation strategy determines the expression of the metric. Note that, in some cases, this approach to the computation of $\gamma_{k}\left(T_{k}\right)$ might not be optimal. The extension to the computation of $\gamma_{k}^{\mathrm{EC}}$ and to TM-FB-DR algorithms is immediate.

It is important to observe that the derivation of a specific algorithm from the general framework presented in Section III depends basically on the assumed observation model. In the following, we consider two channel models: a flat-fading channel and a phase-uncertain channel. We show that the latter can be seen as a particular case of the former, and the detection strategies developed for the first channel extend straightforwardly to the second channel.

\section{A. Flat-Fading Channels: Linear Prediction}

The observation $r_{k}$ is modeled as follows:

$$
r_{k}=c_{k} f_{k}+n_{k}
$$

where $\left\{f_{k}\right\}$ is a sequence of complex random variables, jointly Gaussian, with mean $\eta_{f}$ and circularly symmetric, and the symbol $c_{k}$ is the output symbol of the encoder/modulator FSM. The autocovariance sequence of the fading process follows the isotropic scattering model [36], according to which

$$
C_{f}(n) \triangleq E\left\{f_{k} f_{k-n}^{*}\right\}=J_{0}\left(2 \pi f_{\mathrm{D}} T n\right)
$$

where $J_{0}(\cdot)$ is the zeroth-order Bessel function of the first kind, and $f_{\mathrm{D}} T$ is the normalized fading bandwidth. In the following, we concentrate on Rayleigh fading, with mean $\eta_{f}=0$ and autocorrelation sequence $R_{f}(n)=C_{f}(n)$. In [8], it is shown that for conditionally Gaussian observations, a linear-predictive receiver for sequence detection can be derived, in which a generic metric at epoch $k$ can be exactly computed by considering a prediction order equal to $k-1$. Then it is shown that a finite prediction order usually entails a minimal performance degradation. We now outline the structure of the proposed TM-FB algorithms for this detection strategy.

1) TM-FB-CR Algorithms: In this case, the exponential metric $\gamma_{k}$ used in the recursions is

$$
\begin{aligned}
\gamma_{k}\left(T_{k}\right) & =p\left(r_{k} \mid \boldsymbol{r}_{k-N}^{k-1}, T_{k}\right) P\left\{a_{k}\right\} \\
& =p\left(r_{k} \mid \boldsymbol{r}_{k-N}^{k-1}, \boldsymbol{c}_{k-N}^{k}\right) P\left\{a_{k}\right\} .
\end{aligned}
$$

Since $r_{k}$ is conditionally Gaussian, a reasonable approach would consist of estimating $r_{k}$ based on the previous $N$ observations. By introducing the modified observation ${ }^{5}$

$$
r_{k}^{\prime} \triangleq \frac{r_{k}}{c_{k}}=f_{k}+\frac{n_{k}}{c_{k}}
$$

the exponential metric $\gamma_{k}$ can be expressed as follows:

$$
\gamma_{k}\left(T_{k}\right) \propto \exp \left\{-\frac{\left|c_{k}\right|^{2}}{N_{0}}\right\} \exp \left\{-\frac{\left|r_{k}^{\prime}-\hat{f}_{k}^{F}\right|^{2}}{\epsilon_{k}^{F}}\right\} P\left\{a_{k}\right\}
$$

in which

$$
\hat{f}_{k}^{F} \triangleq \sum_{i=1}^{N} p_{i}^{F} r_{k-i}^{\prime}=\sum_{i=1}^{N} p_{i}^{F} \frac{r_{k-i}}{c_{k-i}}
$$

and

$$
\begin{aligned}
\epsilon_{k}^{F} & \triangleq E\left\{\left|r_{k}^{\prime}-\hat{f}_{k}^{F}\right|^{2} \mid \boldsymbol{c}_{k-N}^{k}\right\} \\
& =E\left\{\left|\frac{r_{k}}{c_{k}}-\sum_{i=1}^{N} p_{i}^{F} \frac{r_{k-i}}{c_{k-i}}\right|^{2} \mid \boldsymbol{c}_{k-N}^{k}\right\}
\end{aligned}
$$

where the sequence of output symbols $c_{k-N}^{k}$ is uniquely determined by the transition $T_{k}$. The prediction coefficients $\left\{p_{i}^{F}\right\}=\left\{p_{i}^{F}\left(S_{k}\right)\right\}$ correspond to the solution of a linear system $\boldsymbol{R}_{S}^{F} \boldsymbol{p}^{F}=\boldsymbol{b}^{F}$, constituted by a set of Yule-Walker equations [38], where $\boldsymbol{R}_{S}^{F}$ is an $N \times N$ matrix with entries $\left[r_{S}^{F}\right]_{i, j}$ given by

$$
\left[r_{S}^{F}\right]_{i, j}= \begin{cases}R_{f}(|i-j|), & \text { if } i \neq j \\ R_{f}(0)+\frac{N_{0}}{\left|c_{k-i}\right|^{2}}, & \text { if } i=j\end{cases}
$$

and $\boldsymbol{b}^{F} \triangleq\left[R_{f}(1), R_{f}(2), \ldots, R_{f}(N)\right]$. For the computation of $\gamma_{k}^{\mathrm{EC}}$ in the case with $L \geq 1$, similar considerations as for the computation of $G_{k}^{C}$, outlined in the following, hold.

2) TM-FB-DR Algorithms: Since $G_{k}^{F}=\gamma_{k}$, the same approach followed in Section V-A.1 and based on forward prediction can be applied to the forward recursion. Specularly, the

\footnotetext{
${ }^{5}$ This is an extension of the approach proposed in [8] and was considered in [37].
} 
exponential metric $G_{k}^{B}$ used in the backward recursion can be expressed as

$$
\begin{aligned}
G_{k}^{B}\left(T_{k+N}\right) \propto \exp & \left\{-\frac{\left|c_{k}\right|^{2}}{N_{0}}\right\} \\
& \cdot \exp \left\{-\frac{\left|r_{k}^{\prime}-\sum_{i=1}^{N} p_{i}^{B} r_{k+i}^{\prime}\right|^{2}}{\epsilon_{k}^{B}}\right\} P\left\{a_{k+N}\right\}
\end{aligned}
$$

where the backward prediction coefficients $\left\{p_{i}^{B}\right\}$ are obtained by solving the Yule-Walker equations, derived by minimizing the mean-square error (MSE)

$$
\epsilon_{k}^{B} \triangleq E\left\{\left|r_{k}^{\prime}-\sum_{i=1}^{N} p_{i}^{B} r_{k+i}^{\prime}\right|^{2} \mid \boldsymbol{c}_{k}^{k+N}\right\}
$$

which lead to the following linear system:

$$
\boldsymbol{R}_{S}^{B} \boldsymbol{p}^{B}=\boldsymbol{b}^{B}
$$

where $\boldsymbol{R}_{S}^{B}=\left(\boldsymbol{R}_{S}^{F}\right)^{T}$ and $\boldsymbol{b}^{B}=\left[R_{f}(-1) R_{f}(-2) \cdots R_{f}(-N+\right.$ 1) $]^{T}$. Due to the symmetry of the fading autocorrelation function $R_{f}(n)$, it is easy to show that $\boldsymbol{p}^{B}=\boldsymbol{p}^{F}$ and $\epsilon_{k}^{B}=\epsilon_{k}^{F}$.

The computation of the exponential metric $G_{k}^{C}$ involved in the completion operation represents the main difference, with respect to the case of TM-FB-CR algorithms. For $L \geq 1$, the chain rule can be applied in the computation of the pdf embedded in $G_{k}^{C}$, obtaining

$$
\begin{aligned}
G_{k}^{C} & \left(S_{k-L}, \boldsymbol{a}_{k-L}^{k+L+N}\right) \\
= & p\left(\boldsymbol{r}_{k-L}^{k+L} \mid \boldsymbol{r}_{k-L-N}^{k-L-1}, \boldsymbol{r}_{k+L+1}^{k+L+N}, \boldsymbol{a}_{k-L-N}^{k+L+N}, \mu_{k}\right) P\left\{\boldsymbol{a}_{k-L}^{k+L+N}\right\} \\
= & {\left[\prod_{i=0}^{2 L} p\left(r_{k+L-i} \mid \boldsymbol{r}_{k-L-N}^{k+L-1-i}, \boldsymbol{r}_{k+L+1}^{k+L+N}, \boldsymbol{a}_{k-L-N}^{k+L+N}, \mu_{k}\right)\right] } \\
& \cdot P\left\{\boldsymbol{a}_{k-L}^{k+L+N}\right\} .
\end{aligned}
$$

Each of the component pdfs in the product inside (40) can be computed by estimating $f_{k+L-i}$ as follows:

$$
\begin{aligned}
\hat{f}_{k+L-i}= & \sum_{\substack{j=1 \\
i=0, \ldots, 2 L}}^{2 L+N-i} p_{j, i}^{C} r_{k+L-1-j}^{\prime}+\sum_{j=1}^{N} p_{j, i}^{C} r_{k+L+j}^{\prime} \\
& i=0, \ldots, 2 \\
&
\end{aligned}
$$

where, for a specific value of $i$, the $2 L+2 N-i$ prediction coefficients $\left\{p_{j, i}^{C}\right\}$ can be obtained by solving a similar set of Yule-Walker equations derived by minimizing the MSE

$$
E\left\{\left|r_{k+L-i}^{\prime}-\hat{f}_{k+L-i}\right|^{2} \mid \boldsymbol{c}_{k-L-N-1-i}^{k+L-2}, \boldsymbol{c}_{k+L+1}^{k+L+N}\right\} .
$$

The computation is a tedious extension of the forward prediction case considered in Section V-A.1. Hence, we limit ourselves to the case with $L=0$. This case does not respect the condition $2 L \geq N$ considered in the general derivation of TM-FB-DR algorithms (or the corresponding condition $2 L \geq Q$ in the case of reduced-state TM-FB-DR algorithms), but the numerical results will show that the performance degradation is minimal, especially if state-reduction techniques are used. In fact, considering the approximation in (12) when $2 L<N$ is equivalent to considering, in the completion operation, a reduced truncation depth corresponding to $2 L$, i.e., lower than $N$. Moreover, in this case, TM-FB-DR algorithms can be given an intuitive explanation. In the two recursions, there is forward and backward prediction, respectively, while in the completion, there is smoothing, since the fading coefficient at epoch $k$ is estimated based on past and future observations [39]. In this case, the fading estimate is

$$
\hat{f}_{k}^{C}=\sum_{i=-1}^{-N} p_{i}^{C} r_{k+i}^{\prime}+\sum_{i=1}^{N} p_{i}^{C} r_{k+i}^{\prime}
$$

and the MSE becomes

$$
\epsilon_{k}^{C}=E\left\{\left|r_{k}^{\prime}-\sum_{i=-1}^{-N} p_{i}^{C} r_{k+i}^{\prime}-\sum_{i=1}^{N} p_{i}^{C} r_{k+i}^{\prime}\right|^{2} \mid \boldsymbol{c}_{k-N}^{k+N}\right\}
$$

where the sequence of coded symbols $\boldsymbol{c}_{k-N}^{k+N}$ is uniquely determined by $\left(\boldsymbol{a}_{k-L-N}^{k+L+N}, \mu_{k}\right)$. By imposing

$$
\frac{\partial \epsilon_{k}^{C}}{\partial p_{i}^{C}}=0 \quad i=-N, \ldots,-1,1, \ldots, N
$$

the following linear system can be derived:

$$
\boldsymbol{R}_{S}^{C} \boldsymbol{p}^{C}=\boldsymbol{b}^{C}
$$

where $\boldsymbol{p}^{C}=\left[p_{-N}^{C}, \ldots, p_{-1}^{C} p_{1}^{C}, \ldots, p_{N}^{C}\right]$ and $\boldsymbol{b}^{C}=$ $\left[R_{f}(N), \ldots, R_{f}(1) R_{f}(1), \ldots, R_{f}(N)\right]$. The $2 N \times 2 N$ system matrix $\boldsymbol{R}_{S}^{C}$ can be written as

$$
\boldsymbol{R}_{S}^{C}=\left[\begin{array}{cc}
\boldsymbol{R}_{S a}^{C} & \boldsymbol{R}_{S b}^{C} \\
\left(\boldsymbol{R}_{S b}^{C}\right)^{T} & \boldsymbol{R}_{S a}^{C}
\end{array}\right]
$$

where $\boldsymbol{R}_{S a}^{C}=\boldsymbol{R}_{S}^{F}$, and the generic element $\left[r_{S b}^{C}\right]_{i, j}$ of the $N \times N$ matrix $\boldsymbol{R}_{S b}^{C}$ has the following expression:

$$
\left[r_{S b}^{C}\right]_{i, j}=R_{f}(N+1-i+j) \quad i, j=1, \ldots, N .
$$

It is easy to recognize that the system matrix $\boldsymbol{R}_{S}^{C}$ is a Hermitian matrix. Hence, the linear system (46) can be solved by considering Cholesky decomposition [40]. After determining the smoothing coefficients which solve (45), the minimum MSE (MMSE) can be written as

$$
\epsilon_{k}^{C}=R_{f}(0)+\frac{N_{0}}{\left|c_{k}\right|^{2}}-\sum_{i=1}^{N}\left(p_{-i}^{C}+p_{i}^{C}\right) R_{f}(i) .
$$

\section{B. Flat-Fading Channels: Noncoherent Detection}

We assume that the observation model is

$$
r_{k}=f e^{j \theta} c_{k}+n_{k}
$$

where $f$ is modeled as a circularly symmetric complex Gaussian random variable with mean $\eta_{f}$ and variance $\sigma_{f}^{2}$, and $\theta$ is modeled as a random variable with uniform distribution in the interval $[0,2 \pi)$, independent of the multiplicative fading coefficient. This model has been considered in [18] in the case of sequence detection, implemented by means of a VA, over slowly 
varying fading channels. According to the considerations made in Section III-B, it is possible to extend this approach to the case of TM-FB-CR algorithms and, further, to TM-FB-DR algorithms. In the following, we derive the general structure of these two classes of algorithms. Observe that, while the derivation in the following holds exactly for the time-invariant fading model in (49), the presence of memory truncation allows the derived algorithms to cope also with a time-varying fading process.

1) TM-FB-CR Algorithms: Assuming that $R_{f}(0)$ and $\left|\eta_{f}\right|$ are known, it is possible to show that [18]

$$
\begin{aligned}
& E_{f, \theta}\left\{p\left(\boldsymbol{r}_{k-N}^{k} \mid \boldsymbol{c}_{k-N}^{k}, f, \theta\right)\right\} I_{0}\left(\frac{2}{N_{0}}\left[\frac{\left|\eta_{f}\right| \sum_{i=0}^{N}\left|c_{k-i}\right|^{2}}{1+\omega \sum_{i=0}^{N}\left|c_{k-i}\right|^{2}}\right]\right) \\
& \propto \frac{1+\omega \sum_{i=0}^{N}\left|c_{k-i}\right|^{2}}{1}\left[\frac{1}{N_{0}}\left[\frac{\omega\left|\sum_{i=0}^{N} r_{k-i} c_{k-i}^{*}\right|^{2}-\left|\eta_{f}\right|^{2} \sum_{i=0}^{N}\left|c_{k-i}\right|^{2}}{1+\omega \sum_{i=0}^{N}\left|c_{k-i}\right|^{2}}\right]\right\}
\end{aligned}
$$

where $\omega \triangleq \sigma_{f}^{2} / N_{0}=\left(R_{f}(0)-\left|\eta_{f}\right|^{2}\right) / N_{0}$. Hence, the metric $\gamma_{k}$ in (28) can be computed. Similarly, the metric $\gamma_{k}^{\mathrm{EC}}$ can be computed as well. By assuming Rayleigh fading $\left(\eta_{f}=0\right)$, large signal-to-noise ratio (SNR) $(\omega \gg 1)$ and/or phase-shift keying (PSK) signaling $\left(\left|c_{k}\right|=1\right)$, the average pdf (50) can be greatly simplified [18]. The derived TM-FB-CR algorithms can also be applied to the case of time-varying fading channels. As the numerical results will show, the performance degradation, with respect to the case of linear prediction (derived from a time-varying fading model), is limited.

2) TM-FB-DR Algorithms: It is straightforward to extend the approach for TM-FB-CR algorithms to the current class of algorithms. The only difference consists of considering proper summation indexes in the used average pdfs, which are formally similar to (50).

\section{Phase-Uncertain Channels: Linear Prediction}

The channel model considered in this case is the following:

$$
r_{k}=e^{j \theta_{k}} c_{k}+n_{k}
$$

where $\left\{n_{k}\right\}$ is an AWGN process, and $\left\{\theta_{k}\right\}$ is a channel-phase process. If the autocorrelation $R_{\theta}(n) \triangleq E\left\{e^{j\left(\theta_{k}-\theta_{k-n}\right)}\right\}$ of the phasor process $\left\{e^{j \theta_{k}}\right\}$ is known, it is then possible to carry out exactly the same derivation as in the case of a flat-fading channel, i.e., in Section V-A, the only difference being the substitution of the autocorrelation $R_{f}(n)$ with the autocorrelation $R_{\theta}(n)$ [41]. In the usual case of a phase process modeled as a Wiener process, $R_{\theta}(n)=e^{-|n| \sigma_{\Delta}^{2}}[41]$.

\section{Phase-Uncertain Channels: Noncoherent Detection}

The observation is expressed as follows:

$$
r_{k}=e^{j \theta} c_{k}+n_{k}
$$

where $\theta$ is modeled as a random variable uniformly distributed in the interval $[0,2 \pi)$. Considering the case of flat-fading channels, as in Section V-B, and imposing $\left|\eta_{f}\right|=1$ and $\sigma_{f}^{2}=0(\omega=$ 0 ), the observation model (49) reduces to (52). It is, therefore, possible to derive the metrics relative to the current channel from those computed for a flat-fading channel.

1) TM-FB-CR Algorithms: Imposing $\omega \rightarrow 0$ and $\left|\eta_{f}\right| \rightarrow 1$, from (50), it is possible to derive

$$
\begin{aligned}
E_{\theta}\left\{p\left(\boldsymbol{r}_{k-N}^{k} \mid \boldsymbol{c}_{k-N}^{k}, \theta\right)\right\} \propto \exp \left\{-\frac{\sum_{i=0}^{N}\left|c_{k-i}\right|^{2}}{N_{0}}\right\} \\
\cdot I_{0}\left(\frac{2}{N_{0}}\left|\sum_{i=0}^{N} r_{k-i} c_{k-i}^{*}\right|\right) .
\end{aligned}
$$

Hence, the exponential metric $\gamma_{k}$ associated with the current channel model can be computed. The exponential metric $\gamma_{k}^{\mathrm{EC}}$ in the extended completion can be similarly computed.

2) TM-FB-DR Algorithms: It is straightforward to derive the exponential metrics used in this class of algorithms by computing proper average pdfs similar to (53).

\section{NUMERICAL RESULTS}

In the following, in order to characterize the proposed TM-FB algorithmic classes, we analyze, through simulations, the performance offered by various iterative detection/decoding schemes. In particular, each TM-FB algorithm is in the min-sum form [35], and any coherent FB algorithm is the standard BCJR algorithm [31]. The extrinsic information generated by each component subdecoder is properly weighted to optimize the performance [42].

\section{A. Rayleigh Fading Channels}

The proposed detection strategies are first compared for transmission of a serially concatenated convolutional code (SCCC) over a Rayleigh flat-fading channel with normalized bandwidth $f_{\mathrm{D}} T=0.01$. The code consists of an outer 4-state, rate-1/2 convolutional code connected through a length-1024 pseudorandom interleaver [43] to an inner 4 -state, rate-1/2 convolutional code. The respective generator matrices are given by

$$
\begin{aligned}
G_{o}(D) & =\left[\begin{array}{ll}
1+D+D^{2} & 1+D^{2}
\end{array}\right] \\
G_{i}(D) & =\left[\begin{array}{ll}
1 & \frac{1+D^{2}}{1+D+D^{2}}
\end{array}\right] .
\end{aligned}
$$

The output symbols are mapped to a quaternary PSK (QPSK) constellation with Gray mapping, resulting in an overall code of spectral efficiency $0.5 \mathrm{~b} / \mathrm{s} / \mathrm{Hz}$. The inner detector/decoder uses a linear predictive TM-FB algorithm, as described in Section V-A. In Fig. 5, the numerical results, in terms of bit-error rate (BER) versus SNR $E_{b} / N_{0}$, are shown, $E_{b}$ being the received energy per information bit and $N_{0}$ the AWGN single-sided power spectral density. Various complexity levels for the inner detector/decoder are considered in terms of truncation depth $N$ and reduced-state parameter $Q$ - the parameter $L$ is set to zero in all cases. The complexities of the TM-FB detection algorithms used in the inner decoder/detector are 


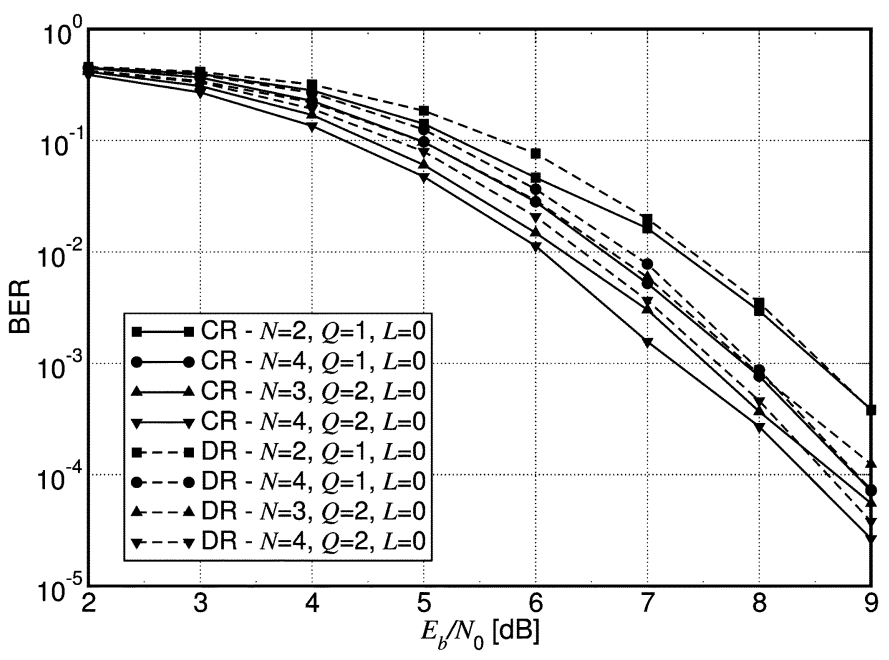

Fig. 5. BER of an SCCC, with inner linear predictive combined detection and decoding, over a Rayleigh flat-fading channel with normalized bandwidth $f_{\mathrm{D}} T=0.01$. In all cases, five decoding iterations are considered.

TABLE III

COMPLEXITy Per TRellis SECTION OF THE TM-FB ALgorithms CONSIDERED IN Fig. 5

\begin{tabular}{c|c|c|c|c|c|c}
\hline Algorithm & $N$ & $Q$ & $L$ & $\zeta_{a}$ & $\zeta_{m}$ & $\zeta_{c}$ \\
\hline \hline CR & 2 & 1 & 0 & 64 & 64 & 18 \\
& 3 & 2 & 0 & 128 & 128 & 34 \\
& 4 & 1 & 0 & 64 & 64 & 18 \\
& 4 & 2 & 0 & 128 & 128 & 34 \\
\hline DR & 2 & 1 & 0 & 96 & 160 & 18 \\
& 3 & 2 & 0 & 320 & 576 & 34 \\
& 4 & 1 & 0 & 96 & 160 & 18 \\
& 4 & 2 & 0 & 320 & 576 & 34 \\
\hline
\end{tabular}

indicated in Table III. Note that in order to apply the results of Tables I and II, one needs to set $M=2$ and $\Omega=4$. In all cases, five decoding iterations are considered. As expected, the complexity of TM-FB-DR algorithms is higher for the same values of $N, Q$, and $L$, than that of TM-FB-CR algorithms. Moreover, TM-FB-CR algorithms perform slightly better (and are characterized by lower complexity) than TM-FB-DR algorithms characterized by the same values of $N$ and $Q$. Increasing the complexity from the case with $(N=2, Q=1)$ to the case with $(N=4, Q=2)$, at a BER equal to $10^{-3}$, there is a performance improvement in terms of SNR of about $1 \mathrm{~dB}$ for TM-FB-CR algorithms, and $1.3 \mathrm{~dB}$ for TM-FB-DR algorithms.

In Fig. 6, the performance obtained using an inner noncoherent decoder in place of the linear predictive decoder is shown. For given values of $N, Q$, and $L$, TM-FB-DR algorithms are more complex than TM-FB-CR algorithms because of the increased complexity of the completion. The complexity levels of all noncoherent TM-FB algorithms used in the inner detector/decoder are shown in Table IV. In particular, for given values of $N$ and $Q$, increasing $L$ from 0 to 1 , one can observe that the performance improvement for TM-FB-DR algorithms is more pronounced than for TM-FB-CR algorithms. In other words, in this case, the extended completion is more beneficial for the algorithmic class with DR. Comparing Fig. 5 with

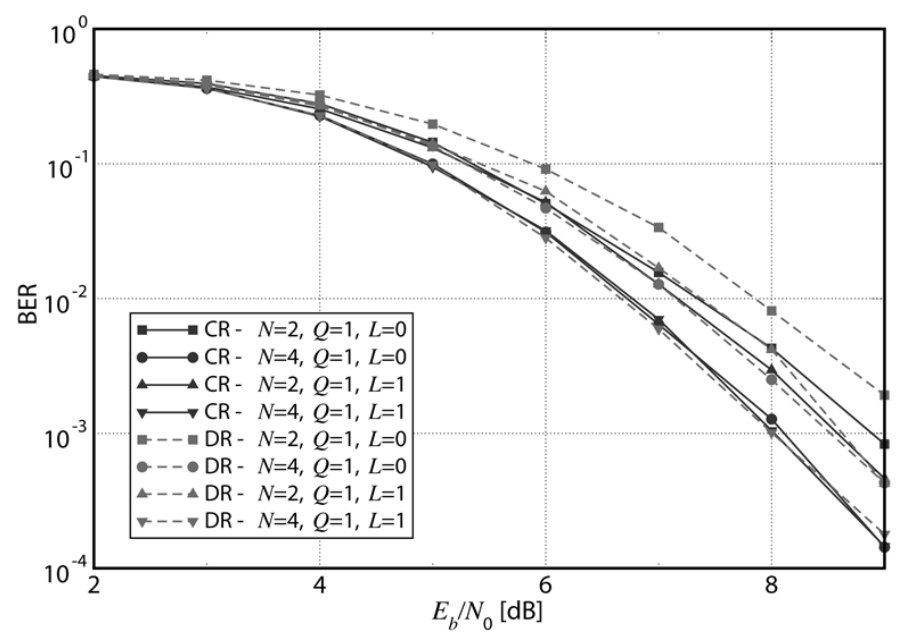

Fig. 6. BER of an SCCC, with inner noncoherent combined detection and decoding, over a Rayleigh flat-fading channel with normalized bandwidth $f_{\mathrm{D}} T=0.01$. In all cases, five decoding iterations are considered.

TABLE IV

COMPleXity PER TRELlis SECTION OF THE TM-FB ALGORITHMS CONSIDERED IN FIG. 6

\begin{tabular}{c|c|c|c|c|c|c}
\hline Algorithm & $N$ & $Q$ & $L$ & $\zeta_{a}$ & $\zeta_{m}$ & $\zeta_{c}$ \\
\hline \hline \multirow{4}{*}{ CR } & 2 & 1 & 0 & 64 & 64 & 18 \\
& 4 & 1 & 0 & 64 & 64 & 18 \\
& 2 & 1 & 1 & 160 & 160 & 18 \\
& 4 & 1 & 1 & 160 & 160 & 18 \\
\hline \multirow{5}{*}{ DR } & 2 & 1 & 0 & 96 & 160 & 18 \\
& 4 & 1 & 0 & 96 & 160 & 18 \\
& 2 & 1 & 1 & 288 & 544 & 18 \\
& 4 & 1 & 1 & 288 & 544 & 18 \\
\hline
\end{tabular}

Fig. 6, it is immediate to recognize that the performance with the two detection strategies, linear predictive and noncoherent, respectively, is similar for the same values of $N, Q$, and $L$ (more precisely, for $N=2, Q=1$, and $L=0$ ).

\section{B. Phase-Uncertain Channels}

In the case of a channel introducing an unknown phase rotation, the proposed detection strategies are compared considering iterative detection of an SCCC. This serial code consists of an outer 4-state, rate-1/2 convolutional code connected through a length-1024 pseudorandom bit interleaver [43] to an inner 4 -state, rate-2/3 convolutional code. The respective generator matrices are given by

$$
G_{o}(D)=\left[\begin{array}{ll}
1 & \frac{1+D^{2}}{1+D+D^{2}}
\end{array}\right] \quad G_{i}(D)=\left[\begin{array}{ccc}
1 & 0 & \frac{1+D^{2}}{1+D+D^{2}} \\
0 & 1 & \frac{1+D}{1+D+D^{2}}
\end{array}\right] .
$$

The output symbols are mapped to an 8-PSK constellation with natural mapping, resulting in an overall code of spectral efficiency $1 \mathrm{~b} / \mathrm{s} / \mathrm{Hz}$. Pilot symbols are introduced with a rate of one pilot every 16 information symbols. Modeling the phase time variations as a Wiener process with incremental standard deviation (over a signaling interval) $\sigma_{\Delta}$, the BER is shown, for increasing phase noise intensity $\sigma_{\Delta}$ and $E_{b} / N_{0}=4 \mathrm{~dB}$, in 


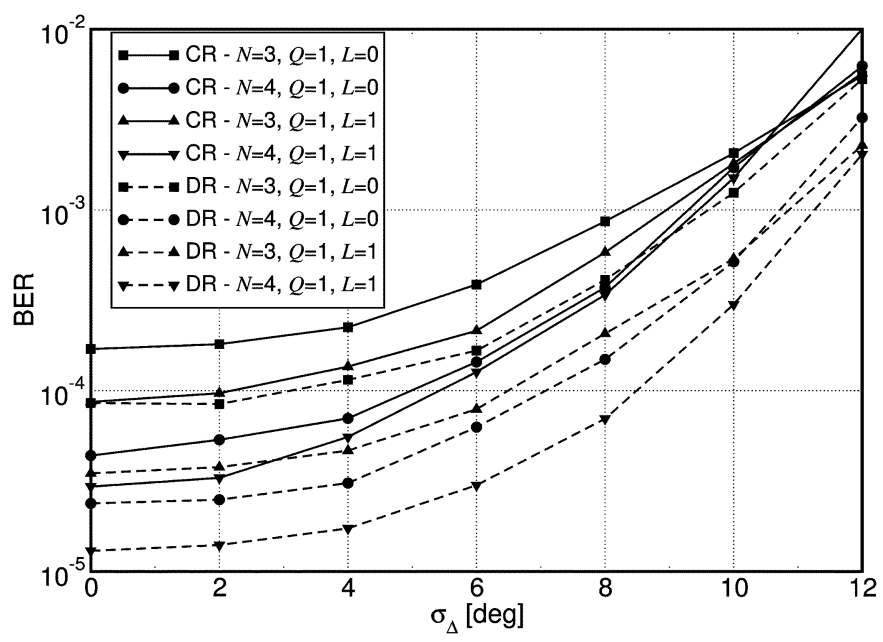

Fig. 7. BER of an SCCC at $E_{b} / N_{0}=4 \mathrm{~dB}$ and five decoding iterations, with inner noncoherent combined detection and decoding, over a phase-uncertain channel for increasing phase-noise standard deviation $\sigma_{\Delta}$.

TABLE $\mathrm{V}$

Complexity Per TRELlis Section of THE TM-FB ALGORITHMS CONSIDERED IN FIG. 7

\begin{tabular}{c|c|c|c|c|c|c}
\hline Algorithm & $N$ & $Q$ & $L$ & $\zeta_{a}$ & $\zeta_{m}$ & $\zeta_{c}$ \\
\hline \hline CR & 3 & 1 & 0 & 384 & 384 & 36 \\
& 4 & 1 & 0 & 384 & 384 & 36 \\
& 3 & 1 & 1 & 4224 & 4224 & 36 \\
& 4 & 1 & 1 & 4224 & 4224 & 36 \\
\hline DR & 3 & 1 & 0 & 1152 & 2176 & 36 \\
& 4 & 1 & 0 & 1152 & 2176 & 36 \\
& 3 & 1 & 1 & 16512 & 32896 & 36 \\
& 4 & 1 & 1 & 16512 & 32896 & 36 \\
\hline
\end{tabular}

Fig. 7. The inner detector/decoder uses the noncoherent TM-FB algorithms derived in Section V-D. In all cases, five decoding iterations and various complexity levels, characterized by the parameters $(N, Q, L)$, are considered. For all algorithms, the corresponding complexity levels at the inner decoder/detector are indicated in Table V. Note that, in order to apply the results of Tables I and II, one needs to set $M=4$ and $\Omega=4$. The performance results obtained considering linear prediction instead of noncoherent detection at the inner detector/decoder (see [41]) are very similar to those shown in Fig. 7. In this case, the complexity of TM-FB-DR algorithms is significantly higher than that of TM-FB-CR algorithms for the same values of $N, Q$, and $L$. This is due to the high cardinality $(M=4)$ of the information symbols at the input of the inner encoder/modulator. In particular, in the cases with $L=1$, i.e., in the case of extended completion, the complexity of TM-FB-DR algorithms becomes intolerably large. Note that, unlike the behavior observed for the communication system considered in Figs. 5 and 6 , in this case, for given values of $N, Q$, and $L$, TM-FB-DR algorithms offer better performance than TM-FB-CR algorithms, but at the price of a much higher complexity. The improvement brought by considering an extended completion is slightly better for TM-FB-DR algorithms.

\section{DISCUSSION}

Based on the numerical results presented in Section VI, we now try to answer the two questions presented at the end of Section I.

1) As anticipated in Section IV-A, for given values of $N$, $Q$, and $L$, TM-FB-DR algorithms have higher complexity than TM-FB-CR algorithms. More precisely, when the complexity is roughly on the same order, TM-FB-CR algorithms perform better (see the results in Section VI-A). However, when the complexity of TM-FB-DR algorithms is significantly larger than that of TM-FB-CR algorithms (for instance, this happens when $M$ is large), then the former algorithms offer better (but not significantly better) performance (see the results in Fig. 7). From a complexity-performance tradeoff, one can conclude that TM-FB-CR algorithms are preferable.

The main advantage of TM-FB-DR algorithms appears to reside in the fact that when state-reduction techniques are used, which is basically unavoidable, to make the complexity of TM-FB algorithms feasible, then the construction of DF in the two recursions can be decoupled [25]. This implies that the two recursions can be started in parallel, and the generation of soft-output reliability values can start as soon as the two recursions "touch" in the center of the observations block. Hence, the decoding latency allowed by the use of reduced-state TM-FB-DR algorithms can potentially be roughly half of that allowed by the use of reduced-state TM-FB-CR algorithms. This potential gain is implementation-dependent, and its simulation-based analysis is beyond the scope of this paper.

Use of extended completion $(L \geq 1)$ is, complexity-wise, very expensive when a trellis section is characterized by many branches (i.e., the cardinality $M$ of the information symbols is high). However, using an extended completion allows improving the performance, leaving the difference $N-Q$ relatively small. The memory requirements to store the DF path associated with the trellis states, being proportional to $N-Q$, are therefore limited. This is a key aspect in the implementation of a turbo decoder, since the largest portion of a chip area is usually occupied by storage blocks [35]. Hence, due to the relatively low cost of current signal-processing blocks, a reduction of memory occupancy can be traded for increased signal-processing capabilities, and this can lead to faster decoding. Finally, we observe that one could possibly devise a CR algorithmic structure where, in the case of state reduction, DF is built independently in the two recursions. In other words, this corresponds to deriving a $\mathrm{CR}$ algorithmic structure where DF is built à la DR. However, since in the exponential metric $\gamma_{k}$, the observation at epoch $k$ is conditioned on past observations, considering DF in the backward recursion might not be very efficient. Novel, more efficient state/complexity reduction techniques might be envisioned for this scenario.

2) Given that a TM detection strategy is considered, the specific strategy (e.g., linear predictive or noncoherent) does not noticeably influence the performance of a particular algorithmic structure. In the case of flat-fading channels, this can be observed, comparing the results in Fig. 5 with those in Fig. 6, and in the case of phase-uncertain channels, this can be observed, comparing the results in Fig. 7 with those in [41]. Basically, the performance seems to depend largely on the truncation depth, i.e., on the value of $N$. In other words, our results 
suggest that, rather than finding new particular detection strategies within the realm of a TM detection framework, one should explore conceptually different approaches to performing detection. For instance, a detection approach based on characterizing the underlying channel through a Markov model leads to excellent results, and seems to offer a better complexity-performance tradeoff than a TM approach [44], [45]. Another very promising approach, based on selecting the decision regions in the received signal space in a clever way, leads to polynomial-complexity detection algorithms [46], [47].

\section{CONCLUSIONS}

A general framework for trellis-based TM detection has been derived. It has been shown that TM sequence detection algorithms are systematically related to TM symbol detection algorithms. Two main classes of TM-FB algorithms have been derived, with CR and DR, respectively. In both cases, it has been shown that an extended completion can be considered. A simple complexity analysis has shown that, for given values of main algorithmic parameters $(N, Q, L)$, TM-FB-DR algorithms are more computationally intense than TM-FB-CR algorithms, and the performance improvement, if any, is not dramatic. However, TM-FB-DR algorithms are attractive from an implementation standpoint, since, for a given performance, the decoding latency can be lower than for TM-FB-CR algorithms. The proposed framework has been applied to two stochastic channels, a flat-fading channel and a phase-uncertain channel, considering linear predictive and noncoherent detection. In all cases, the performance seems to ultimately depend on the truncation depth, while different detection strategies entail minor performance differences.

\section{ACKNOWLEDGMENT}

The authors would like to thank the Editor, Prof. R. D. Wesel, for his extremely detailed comments, which significantly improved the quality of this paper. In particular, the Editor suggested the extension of the CR architecture to include extended completion, which made the overall paper more insightful and complete. The reviewers are also gratefully acknowledged for several useful comments which improved the quality of the paper.

\section{REFERENCES}

[1] H. L. VanTrees, Detection, Estimation, and Modulation Theory-Part I. New York: Wiley, 1968.

[2] R. Raheli, A. Polydoros, and C. Tzou, "Per-survivor processing: A general approach to MLSE in uncertain environments," IEEE Trans. Commun., vol. 43, no. 2-4, pp. 354-364, Feb.-Apr. 1995.

[3] K. M. Chugg and A. Polydoros, "MLSE for an unknown channel-Part I: Optimality considerations," IEEE Trans. Commun., vol. 44, no. 7, pp. 836-846, Jul. 1996.

[4] - "MLSE for an unknown channel—Part II: Tracking performance," IEEE Trans. Commun., vol. 44, no. 8, pp. 949-958, Aug. 1996.

[5] A. Anastasopoulos and K. M. Chugg, "Adaptive soft-input soft-output algorithms for iterative detection with parametric uncertainty," IEEE Trans. Commun., vol. 48, no. 10, pp. 1638-1649, Oct. 2000.

[6] K. M. Chugg and A. Polydoros, "Adaptive iterative detection for phase tracking in turbo coded systems," IEEE Trans. Commun., vol. 49, no. 12, pp. 2135-2144, Dec. 2001.

[7] J. Makhoul, "Linear prediction: A tutorial review," Proc. IEEE, vol. 63, no. 4, pp. 561-580, Apr. 1975.
[8] J. Lodge and M. Moher, "Maximum-likelihood estimation of CPM signals transmitted over Rayleigh flat fading channels," IEEE Trans. Commun., vol. 38, no. 6, pp. 787-794, Jun. 1990.

[9] D. Makrakis, P. T. Mathiopoulos, and D. Bouras, "Optimal decoding of coded PSK and QAM signals in correlated fast fading channels and AWGN: A combined envelope, multiple differential, and coherent detection approach," IEEE Trans. Commun., vol. 42, no. 1, pp. 63-75, Jan. 1994.

[10] X. Yu and S. Pasupathy, "Innovations-based MLSE for Rayleigh fading channels," IEEE Trans. Commun., vol. 43, no. 2-4, pp. 1534-1544, Feb.-Apr. 1995.

[11] G. M. Vitetta and D. P. Taylor, "Maximum-likelihood decoding of uncoded and coded PSK signal sequences transmitted over Rayleigh flatfading channels," IEEE Trans. Commun., vol. 43, no. 11, pp. 2750-2758, Nov. 1995.

[12] G. Vitetta, D. Taylor, and U. Mengali, "Double filtering receivers for PSK signals transmitted over Rayleigh frequency flat-fading channels," IEEE Trans. Commun., vol. 44, no. 6, pp. 686-695, Jun. 1996.

[13] G. Vitetta, U. Mengali, and D. Taylor, "Double-filter differential detection of PSK signals transmitted over linearly time-selective Rayleigh fading channels," IEEE Trans. Commun., vol. 47, no. 2, pp. 239-247, Feb. 1999.

[14] M. J. Gertsman and J. H. Lodge, "Symbol-by-symbol MAP demodulation of CPM and PSK signals on Rayleigh flat-fading channels," IEEE Trans. Commun., vol. 45, no. 7, pp. 788-799, Jul. 1997.

[15] I. D. Marsland and P. T. Mathiopoulos, "Multiple differential detection of parallel concatenated convolutional (turbo) codes in correlated fast Rayleigh fading," IEEE J. Sel. Areas Commun., vol. 16, no. 2, pp. 265-275, Feb. 1998.

[16] M. C. Valenti and B. D. Woerner, "Iterative channel estimation and decoding of pilot-symbol-assisted turbo codes over flat-fading channels," IEEE J. Sel. Areas Commun., vol. 19, no. 9, pp. 1697-1705, Sep. 2001.

[17] A. Hansson, K. M. Chugg, and T. Aulin, "On forward-adaptive versus forward/backward-adaptive SISO algorithms for Rayleigh fading channels," IEEE Commun. Lett., vol. 5, no. 12, pp. 477-479, Dec. 2001.

[18] G. Colavolpe and R. Raheli, "Noncoherent sequence detection in frequency-nonselective slowly fading channels," IEEE J. Sel. Areas Commun., vol. 18, no. 11, pp. 2302-2311, Nov. 2000.

[19] - "Noncoherent sequence detection," IEEE Trans. Commun., vol. 47, no. 9, pp. 1376-1385, Sep. 1999.

[20] G. Colavolpe, G. Ferrari, and R. Raheli, "Noncoherent iterative (turbo) detection," IEEE Trans. Commun., vol. 48, no. 9, pp. 1488-1498, Sep. 2000.

[21] G. Ferrari, G. Colavolpe, and R. Raheli, "Noncoherent iterative decoding of spectrally efficient coded modulations," Ann. Telecommun., vol. 56, pp. 409-421, Jul./Aug. 2001.

[22] I. D. Marsland and P. T. Mathiopoulos, "On the performance of iterative noncoherent detection of coded $M$-PSK signals," IEEE Trans. Commun., vol. 48, no. 4, pp. 588-596, Apr. 2000.

[23] A. Kavćić and J. M. F. Moura, "Correlation-sensitive adaptive sequence detection," IEEE Trans. Magn., vol. 34, no. 5, pp. 763-771, May 1998.

[24] G. Colavolpe, G. Ferrari, and R. Raheli, "Reduced-state BCJR-type algorithms," IEEE J. Sel. Areas Commun., vol. 19, no. 5, pp. 848-859, May 2001.

[25] P. Thiennviboon, G. Ferrari, and K. M. Chugg, "Generalized trellisbased reduced-state soft-input/soft-output algorithms," in Proc. IEEE Int. Conf. Commun., New York, NY, Apr. 2002, pp. 1667-1671.

[26] D. Bokolamulla, A. Hansson, and T. Aulin, "Low-complexity iterative detection based on bidirectional trellis search," in Proc. IEEE Symp. Inf. Theory, Yokohama, Japan, Jul. 2003, pp. 396-396.

[27] B. E. Rimoldi, "A decomposition approach to CPM," IEEE Trans. Inf Theory, vol. 34, no. 3, pp. 260-270, Mar. 1988.

[28] G. Ferrari, G. Colavolpe, and R. Raheli, "A unified framework for finitememory detection,” IEEE J. Sel. Areas Commun., pp. -, Sep. 2005.

[29] S. Benedetto, D. Divsalar, G. Montorsi, and F. Pollara, "A soft-input soft-output APP module for iterative decoding of concatenated codes," IEEE Commun. Lett., vol. 1, no. 1, pp. 22-24, Jan. 1997.

[30] K. M. Chugg, "The condition for the applicability of the Viterb algorithm with implications for fading channel MLSD," IEEE Trans. Commun., vol. 46, no. 9, pp. 1112-1116, Sep. 1998.

[31] L. R. Bahl, J. Cocke, F. Jelinek, and J. Raviv, "Optimal decoding of linear codes for minimizing symbol error rate," IEEE Trans. Inf. Theory, vol. IT-20, no. 3, pp. 284-287, Mar. 1974.

[32] G. Ferrari, "On iterative detection for channels with memory," Ph.D. dissertation, Univ. Parma, Parma, Italy, 2001.

[33] G. D. Forney, Jr., "The Viterbi algorithm," Proc. IEEE, vol. 61, no. 3 , pp. 268-278, Mar. 1973. 
[34] P. Robertson, E. Villebrun, and P. Hoeher, "Optimal and sub-optimal maximum a posteriori algorithms suitable for turbo decoding," Eur. Trans. Telecommun., vol. 8, no. 2, pp. 119-125, Mar./Apr. 1997.

[35] K. M. Chugg, A. Anastasopoulos, and X. Chen, Iterative Detection: Adaptivity, Complexity Reduction, and Applications. Norwell, MA: Kluwer, 2001.

[36] W. C. Jakes, Microwave Mobile Communications. New York: Wiley, 1974.

[37] G. Colavolpe, P. Castoldi, and R. Raheli, "Linear predictive receivers for fading channels," IEE Electron. Lett., vol. 34, no. 6, pp. 1289-1290, Jun. 1998.

[38] A. Papoulis, Probability, Random Variables and Stochastic Processes. New York: McGraw-Hill, 1991.

[39] J. M. Mendel, Lessons in Estimation Theory for Signal Processing, Communications, and Control. Upper Saddle River, NJ: Prentice-Hall, 1995.

[40] G. H. Golub and C. F. Van Loan, Matrix Computations, 3rd ed. Baltimore, MD: Johns Hopkins Univ. Press, 1996.

[41] G. Ferrari, G. Colavolpe, and R. Raheli, "Linear predictive receivers for phase uncertain channels," in Proc. Int. Symp. Signal Process. Appl., vol. 1, Paris, France, Jul. 2003, pp. 301-304.

[42] G. Colavolpe, G. Ferrari, and R. Raheli, "Extrinsic information in iterative decoding: A unified view," IEEE Trans. Commun., vol. 49, no. 12, pp. 2088-2094, Dec. 2001

[43] C. Berrou and A. Glavieux, "Near-optimum error-correcting coding and decoding: Turbo codes," IEEE Trans. Commun., vol. 44, no. 10, pp. 1261-1271, Oct. 1996

[44] M. Peleg, S. Shamai (Shitz), and S. Galán, "Iterative decoding for coded noncoherent MPSK communications over phase-noisy AWGN channel," IEE Proc. Commun., vol. 147, pp. 87-95, Apr. 2000.

[45] C. Komninakis and R. D. Wesel, "Joint iterative channel estimation and decoding in flat correlated Rayleigh fading," IEEE J. Sel. Areas Commun., vol. 19, no. 9, pp. 1706-1717, Sep. 2001

[46] J. K. M. Mackenthun, "A fast algorithm for multiple-symbol differential detection of MPSK," IEEE Trans. Commun., vol. 33, no. 2-4, pp. 1471-1474, Feb.-Apr. 1994.

[47] I. Motedayen-Aval and A. Anastasopoulos, "Polynomial-complexity noncoherent symbol-by-symbol detection with application to adaptive iterative decoding of turbo-like codes," IEEE Trans. Commun., vol. 51, no. 2, pp. 197-207, Feb. 2003.

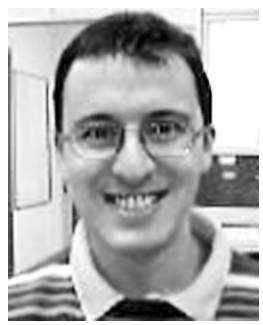

Gianluigi Ferrari (S'97-M'03) was born in Parma, Italy, in November 1974. He received the "Laurea" degree (five-year program summa cum laude) and the Ph.D. degree, both in electrical engineering, from the University of Parma, Parma, Italy, in 1998 and 2002, respectively.

From July 2000 to December 2001, he was a Visiting Scholar at the Communication Sciences Institute, University of Southern California, Los Angeles, CA. Since 2002, he has been a Research Professor with the Department of Information Engineering, University of Parma. Between 2002 and 2004, he visited, as a Research Associate, the Electrical and Computer Engineering Department, Carnegie Mellon University, Pittsburgh, PA, several times. He has published more than 50 papers in leading international conferences and journals. He is coauthor of the books Detection Algorithms for Wireless Communications, with Applications to Wired and Storage Systems (Chichester: U.K., 2004), Ad Hoc Wireless Networks: A Communication-Theoretic Perspective (Chichester, U.K.: Wiley, 2006), and Teoria della Probabilité Variabili Aleatorie, Con Applicazioni (Milan, Italy: McGraw-Hill, 2005). He is listed in Who's Who in the World (New Providence, NJ: Marquis Who's Who, 2005), and in 2000 Outstanding Intellectuals of the 21st Century (Cambridgeshire, U.K.: International Biographical Centre, 2005). His research interests include digital communication systems design, adaptive signal processing (with particular emphasis on iterative detection techniques for channels with memory), information theory and ad hoc wireless networking. He acts as a frequent reviewer for many international journals and conferences.

Dr. Ferrari acts also as a Technical Program Member for several international conferences, including the IEEE International Conference on Communcations, Seoul, Korea, 2005, and the IEEE International Conference on Wireless Networks, Communications, and Mobile Computing, Maui, HI, 2005.

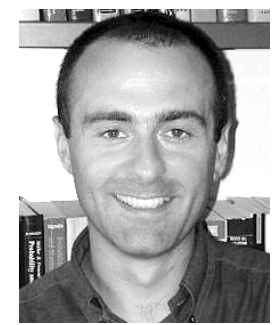

Giulio Colavolpe (S'96-A'00) was born in Cosenza, Italy, in 1969. He received the Dr. Ing. degree (cum laude) in telecommunication engineering from the University of Pisa, Pisa, Italy, in 1994 and the $\mathrm{Ph} . \mathrm{D}$. degree in information technology from the University of Parma, Parma, Italy, in 1998.

Since 1997, he has been with the University of Parma, where he is now an Associate Professor of Telecommunications. In 2000, he was a Visiting Scientist with the Institut Eurécom, Valbonne, France. His main research interests include digital transmission theory, channel coding, and signal processing. His research activity has led to more than 70 scientific publications in leading international journals and conference proceedings and several industrial patents. He is also coauthor of the book Detection Algorithms for Wireless Communications, with Applications to Wired and Storage Systems (Chichester, U.K.: Wiley, 2004).

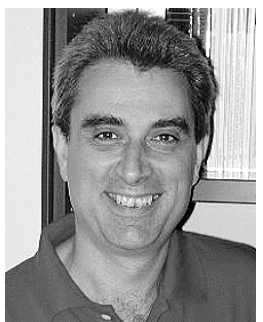

Riccardo Raheli (M'87) received the Dr.Ing. degree (Laurea) in electrical engineering (summa cum laude) from the University of Pisa, Pisa, Italy, in 1983, the M.S. degree in electrical and computer engineering from the University of Massachusetts, Amherst, in 1986, and the Ph.D. degree (Perfezionamento) in electrical engineering (summa cum laude) from the Scuola Superiore di Studi Universitari e di Perfezionamento (now "S. Anna"), Pisa, Italy, in 1987.

From 1986 to 1988, he was with Siemens Telecomunicazioni, Cassina de' Pecchi (Milan), Italy. From 1988 to 1991, he was a Research Professor at the Scuola Superiore di Studi Universitari e di Perfezionamento S. Anna, Pisa, Italy. In 1990, he was a Visiting Assistant Professor at the University of Southern California, Los Angeles. Since 1991, he has been with the University of Parma, Parma, Italy, where he is currently a Professor of Communications Engineering. His scientific interests are in the general area of statistical communication theory, with application to wireless, wired and storage systems, and special attention to data detection in uncertain environments, iterative information processing and adaptive algorithms for communications. His research activity has lead to numerous scientific publications in leading international journals and conference proceedings, as well as a few industrial patents. In 1990, he conceived (with A. Polydoros) the principle of per-survivor processing. He is coauthor of the book Detection Algorithms for Wireless Communications, with Applications to Wired and Storage Systems (Chichester, U.K.: Wiley, 2004).

Dr. Raheli served on the Editorial Board of the IEEE TRANSACTIONS ON COMMUNICATIONS as an Editor for Detection, Equalization, and Coding from 1999 to 2003. He is currently serving as a Guest Editor of the IEEE JOURNAL ON SElECted AREAS IN COMmunications, Special Issue on Differential and Noncoherent Wireless Communications. Since 2003, he has been on the Editorial Board of the European Transactions On Telecommunications as an Editor for Communication Theory. 